\title{
The Habenular Receptor GPR151 Regulates Addiction Vulnerability Across Drug Classes
}

Beatriz Antolin-Fontes ${ }^{1}$, Kun Li ${ }^{1}$, Jessica L. Ables ${ }^{1,2,3}$, Michael H. Riad ${ }^{1}$, Andreas Görlich ${ }^{1}$ Maya Williams ${ }^{2}$, Cuidong Wang ${ }^{1}$, Sylvia M. Lipford ${ }^{1}$, Maria Dao $^{2}$, Henrik Molina ${ }^{5}$, Jianxi Liu ${ }^{4}$, Paul J. Kenny ${ }^{2,4}$ and Ines Ibañez-Tallon ${ }^{1 *}$

${ }^{1}$ Laboratory of Molecular Biology, The Rockefeller University, 1230 York Avenue, New York, NY 10065, USA.

${ }^{2}$ Nash Family Department of Neuroscience, Icahn School of Medicine at Mount Sinai, One Gustave L. Levy Place, New York, NY 10029-6574, USA.

${ }^{3}$ Department of Psychiatry, Icahn School of Medicine at Mount Sinai, One Gustave L. Levy Place, New York, NY 10029-6574, USA.

${ }^{4}$ Drug Discovery Institute Department of Pharmacology \& Systems Therapeutics, Icahn School of Medicine at Mount Sinai, One Gustave L. Levy Place, New York, NY 10029-6574, USA.

${ }^{5}$ Proteomics Resource Center, The Rockefeller University, 1230 York Avenue, New York, NY 10065, USA.

*Corresponding Author and Lead Contact: iibanez@rockefeller.edu 


\section{SUMMARY}

The habenula controls the addictive properties of nicotine but also densely expresses opioid and cannabinoid receptors. As such, identification of strategies to manipulate habenular activity may yield new approaches to treat substance use disorders. Here we show that GPR151, an orphan G protein-coupled receptor (GPCR) highly enriched in the habenula of humans and rodents plays a critical role in regulating habenular function and behavioral responses to addictive drugs. We show that GPR151 is expressed on axonal and presynaptic membranes and synaptic vesicles, and regulates synaptic fidelity and plasticity. We find that GPR151 associates with synaptic components controlling vesicle release and ion transport and couples to the G-alpha inhibitory protein $\mathrm{G}_{\alpha_{01}}$ to reduce cAMP levels. Stable cell lines expressing GPR151 confirm that it signals via Gi/o and are amenable to ligand screens. Gpr151 null mice show diminished behavioral responses to nicotine, and self-administer greater quantities of the drug, phenotypes rescued by viral re-expression of Gpr151 in the habenula. Gpr151 null mice are also insensitive to the behavioral actions of morphine and cannabinoids. These data identify GPR151 as a critical modulator of habenular function that controls addiction vulnerability across different drug classes. 


\section{Highlights}

- Habenula neurons are enriched in nicotinic, opioid, cannabinoid and GPR151 receptors

- GPR151 modulates synaptic fidelity and release probability at habenular terminals.

- Habenular GPR151 plays a role in drug abuse and food intake/weight control

- GPR151 couples to the G-alpha inhibitory protein $\mathrm{G} \alpha{ }_{01}$ to reduce cAMP levels.

\section{eTOC Blurb}

Antolin-Fontes at al. identify a G protein-coupled receptor, GPR151, which is highly enriched in human habenular neurons. These neurons are primarily enriched with nicotinic, opioid and cannabinoid receptors. We find that GPR151 modulates habenular synaptic vesicle release probability and behavioral responses to these drugs of abuse.

\section{INTRODUCTION}

A fundamental role for the habenula in the control of impulsive or compulsive behaviors, and in the devaluation of reward by delay and effort, has been supported strongly by several recent studies (Kobayashi et al., 2013; Lammel et al., 2012; Proulx et al., 2014). The conservation of this ancient structure across vertebrate evolution, and the many behavioral abnormalities evident in animals carrying habenular lesions (Agetsuma et al., 2010; Kobayashi et al., 2013), further support the crucial roles of the habenula in processing reward-related and aversive signals. However, it has become evident that alterations in the functions of this pathway are also associated with psychiatric disorders including obsessive 
compulsive disorder (Proulx et al., 2014) and drug addiction (Antolin-Fontes et al., 2015; McLaughlin et al., 2017).

The global impact of substance abuse and addiction disorders on health and economy is well documented. Currently, about 1.3 billion people consume tobacco and 185 million people use psychoactive drugs (WHO), while the Department of Health and Human services estimates that 2.1 million people in the US have an opioid use disorder, while another 11.2 million misuse opioids. Irrespective of the type of drug, addiction is a chronic relapsing disorder characterized by compulsive drug seeking, escalation of intake, and development of affective and physical symptoms of withdrawal upon abrupt discontinuation or decrease in intake (Koob and Volkow, 2016). Animal studies and human imaging analyses have revealed that many psychoactive drugs act on the mesocorticolimbic dopamine system, a circuit that appears to be common to the rewarding effects of some drugs of abuse, as well as other reinforcing natural behaviors such as eating, thirst, gambling and sexual drive (Lammel et al., 2014; Lammel et al., 2012; Nutt et al., 2015).However recent findings challenge the view that DA release has an exclusive role in reward processing and that stress, and aversive stimuli activate and remodel partially overlapping networks within the mesocorticolimbic dopamine (DA) system (Ostroumov and Dani, 2018). More recently, other structures have been implicated in reward and aversion, including the habenula (Antolin-Fontes et al., 2015; Boulos et al., 2019; Mathis and Kenny, 2018). Increasing evidence suggests that the degree of sensitivity to both the rewarding and aversive aspects of an addictive drug and the severity of the withdrawal after discontinuing its use contribute to the addiction process (Koob and Volkow, 2016). Understanding the mechanisms of these processes may reveal novel insights into the mechanics of the disorder and identify new targets for medications development. 
The medial habenula $(\mathrm{MHb})$ and its major projection site, the interpeduncular nucleus (IPN) are highly enriched in nicotinic acetylcholine receptors (nAChRs) (Ables et al., 2017; Changeux, 2010; Gorlich et al., 2013; Shih et al., 2014) and are key in the control of nicotine intake, aversion, withdrawal and relapse (Fowler et al., 2011; Frahm et al., 2011; Gorlich et al., 2013; Salas et al., 2009). The MHb also densely expresses mu-opioid (MOR) and cannabinoid $1(\mathrm{CB} 1 \mathrm{R})$ receptors. It has been implicated in morphine dependence and withdrawal (Neugebauer et al., 2013), aversion to MOR blockade (Boulos et al., 2019), aversive memories mediated by CB1R (Soria-Gomez et al., 2015) and depression induced by decreased habenular CB1R during nicotine abstinence (Simonnet et al., 2017). Furthermore, it has been shown that chronic exposure to D-amphetamine, methamphetamine, MDMA, cocaine, or nicotine can induce degeneration of the fasciculus retroflexus (FR), the main output tract of the habenula (Velasquez et al., 2014). The finding that this descending pathway is compromised following drug binges has implications not only for theories of drug addiction but also for psychosis in general (Ellison, 2002). Thus, the emerging picture is that the Hb-IPN pathway acts as an inhibitory motivational signal that limits drug intake (Fowler et al., 2011), and that alterations in the functioning of this pathway by drug consumption may contribute to many aspects of addiction.

Here we investigated GPR151, an orphan GPCR with remarkably selective expression in habenular axonal projections. We found that GPR151 is highly conserved in the human $\mathrm{Hb}$ IPN circuit and specifically localizes to axonal and presynaptic membranes and synaptic vesicles (SV). GPR151 partially overlaps with a3ß4-containing $\mathrm{nAChRs,} \mathrm{MOR}$ and CB1R receptors at habenular terminals in the IPN. We observed reduced behavioral responses to drugs that act at these receptors in Gpr151-knockout (KO) mice. Furthermore, Gpr151-KO mice intravenously self-administered much higher amounts of nicotine than their wildtype (WT) counterparts, consistent with a role for GPR151 in regulating the stimulatory effects of 
nicotine on habenular aversion circuits. Mass spectrometry identified SV proteins, presynaptic ATPases and the G-proteins Gao1 and Gß1 as GPR151 interacting proteins, indicating that GPR151 couples to the G-alpha inhibitory pathway to reduce cAMP. In the absence of a ligand we employed optogenetics to stimulate $\mathrm{MHb}$ terminals and found increased synaptic fidelity rates and smaller evoked EPSCs in Gpr151-KO mice. Taken together, our data reveal a key role for GPR151, and the MHb-IPN circuit more broadly, in regulating the sensitivity and aversion to addictive drugs across class, and suggest that small molecule modulators of GPR151 activity may represent an entirely new class of addiction therapeutics.

\section{RESULTS}

\section{GPR151 is specifically co-expressed in the habenula-IPN pathway with nicotinic,} opioid and cannabinoid receptors.

GPR151 expression is remarkably conserved and restricted to the habenula-IPN tract in the brains of zebrafish, mice and rats (Broms et al., 2015). Most GPR151-expressing cells are concentrated in the $\mathrm{Hb}$ (mostly in the $\mathrm{MHb}$ and few scattered cells in the LHb (Broms et al., 2015; Kobayashi et al., 2013). Notably, GPR151 is not present in the soma of habenular neurons, but along their axonal projections that comprise the FR and at terminals in the IPN (Figure 1A-D). To investigate whether the pattern of GPR151 expression was also conserved in humans, we obtained post-mortem adult human brain samples containing part of the diencephalon and midbrain (Figure 1E-O). Immunostaining analyses showed that GPR151 specifically labeled habenular projections arising in the MHb and LHb (Figure 1G-I). We detected very strong GPR151 signal along the FR in horizontal human brain sections (Figure $1 \mathrm{~J}-\mathrm{L}$ ), as well as in the IPN (Figure 1M-O) where $\mathrm{MHb}$ projections terminate. The specific immunoreactivity of human $\mathrm{Hb}$ and IPN to GPR151 antibody is identical to the pattern we observed in rodents and zebrafish (Broms et al., 2015). Western blot analyses in GPR151 
transfected cells, WT mice and human IPN samples revealed two bands corresponding to GPR151: one of the expected size at $46 \mathrm{kDa}$ and an additional higher molecular band $(\approx 53$ kDa) suggesting posttranslational modifications (Figure 1P). These results show that GPR151 is specifically expressed in the Hb-IPN axonal tract in humans, suggesting that its function is likely to be conserved across vertebrates.

To obtain a quantitative list of highly translated mRNAs corresponding to receptors expressed in the same neurons as Gpr151, we employed Chat-EGFP-L10a transgenic mice for Translating Ribosome Affinity Purification (TRAP) analysis (Doyle et al., 2008). Six biological replicates were collected from the habenulae of Chat-EGFP-L10a mice and the resulting INPUT and Immunoprecipitated (IP) mRNAs were analyzed by RNA-Seq (Figure 1Q and Table S1). The high enrichment of Gpr151 in the TRAP IP (Figure S1A, S1B, S2A and Table S1) is consistent with its expression in habenular cholinergic neurons, and indicates that other receptors co-enriched in the IP fraction are also enriched in habenular neurons. These analyses demonstrate high co-enrichment of Gpr151 with Chrna3 and Chrnb4, and significant overlap with Oprm1 and Cnr1 mRNAs in the Chat MHb population (Figure 1Q and S2A).

To confirm the TRAP results, we performed immunofluorescence analyses in Chrna3/eGFP mice, which label $\alpha 3 \beta 4$ nAChR positive neurons in the brain (Frahm et al., 2011). Intense nAChRa3-eGFP expression was evident in MHb neurons and their projections to the IPN (Figure S3B, S3E) and demonstrated considerable overlap with GPR151 (co-localization index: 0.91) in the central and rostral part of the IPN (IPC and IPR) (Figure 1S and Figure S3E-F). Similarly, co-immunostaining of GPR151 with the $\mu$-opioid receptor (MOR) antibody showed intense colocalization in axonal terminals in the IPR (colocalization index: 0.97) (Figure 1T and Figure S3G-H) and partial overlap in the lateral part of the MHb (Figure S3C). 
Double immunostaining of GPR151 and the cannabinoid receptor 1 (CB1R) revealed intense costaining in habenular axons terminals in the IPR (colocalization index: 0.86) (Figure $1 \mathrm{~V}$ and Figure S3I-J) and low expression in habenular cell bodies (Figure S3D). Single stains of MOR and CB1R (Figure S3) are consistent with their reported patterns in habenula and IPN (Gardon et al., 2014; Soria-Gomez et al., 2015). Importantly, gene deletion of Gpr151 in mice (Gpr151-KO mice) does not influence the levels of translated mRNAs of Oprm1 and Cnr1 in $\mathrm{MHb}$ neurons quantified by TRAP (Figure $1 \mathrm{Q}$ ), nor the expression of MOR and CB1R at habenular terminals (Figure S4), indicating that GPR151 expression does not modulate expression of these receptors localized at the same habenular terminals nor induce adaptive responses in their expression in the mutant mice. Together, the TRAP and IHC results establish that GPR151 is highly enriched in habenular projections in the IPN that also express $\alpha 3 \beta 4$ nAChRs, MOR and CB1R, and raise the possibility that GPR151 may influence the actions of of nicotine, morphine and cannabinoids in the habenula and thereby regulate behavioral responses to these drugs.

\section{Reduced sensitivity to nicotine, morphine and the cannabinoid agonist ACEA in Gpr151-KO mice.}

Given the high-degree of conservation of GPR151 between rodent and human brains and the co-expression of GPR151 with receptors for multiple drugs of abuse, we examined the behavioral responses in mice lacking this receptor. This is important because the findings could be potentially relevant to human disorders with reward-related abnormalities, such as affective and addictive disorders. We first tested Gpr151-KO mice at baseline conditions and found no differences in locomotor activity, anxiety-like behaviors measured by the elevated plus maze, sensorimotor gating analyzed by the prepulse inhibition test, and anhedonic-like behavior measured by the preference of sucrose consumed in a two-bottle choice experiment 
(Figure S5A-D), suggesting that GPR151 does not regulate affective-related behaviors at baseline.

We next examined the behavioral responses of Gpr151-KO mice to nicotine, morphine and a synthetic cannabinoid agonist. We assayed locomotor activity after an acute nicotine challenge, which reflects the sensitivity of an individual to nicotine (Clarke and Kumar, 1983). Baseline activity of Gpr151-KO mice was similar to WT (min 0 in Figure 2A, day 0 in Figure 2B, saline in Figure 2C and Figure S5A). However, acute nicotine-induced hypolocomotion was significantly less prominent in Gpr151-KO mice (min 5, 10 in Figure 2A, day 1 in Figure 2B), indicating that Gpr151-KO mice have reduced sensitivity to the motor-depressing property of nicotine. While both WT and KO mice displayed similar nicotine-induced hypolocomotion at the $0.35 \mathrm{mg} / \mathrm{kg}$ dose, unlike the WT mice, Gpr151-KO hypolocomotion was not further increased at 0.65 and $1.5 \mathrm{mg} / \mathrm{kg}$ nicotine (Figure 2C). In addition, daily injections of nicotine induced tolerance to the hypolocomotor effect of nicotine in WT mice after 9 days of treatment, but Gpr151-KO mice did not demonstrate an adaptive response to repeated injection of nicotine (Figure 2B, day 9-11). These results show that Gpr151-KO mice are less sensitive to high doses of nicotine and do not show behavioral plasticity in response to repeated exposures of nicotine.

Next, to rule out a developmental effect caused by the lack of Gpr151 as the reason for the altered sensitivity to nicotine observed in Gpr151-KO mice, we knocked downed and rescued Gpr151 in the habenula of WT and Gpr151-KO mice, respectively. We tested several different shRNAs against Gpr151 in HEK293T cells and determined that shRNA-3 successfully reduced GPR151 expression by Western blot (Figure S6A-B), and we chose it for in vivo analysis. Further, we determined that AAV2/1 yielded the highest level of expression in $\mathrm{MHb}$ neurons compared to other serotypes, and generated AAVs containing 
the shRNA or a scramble-shRNA as a control to knock-down Gpr151 in WT mice and an AAV containing CAG-Gpr151 for the rescue experiments in Gpr151-KO mice. Viruses were injected bilaterally into the MHb and presence or absence of GPR151 expression in the habenula, along the FR and at the IPN was validated in the injected mice by immunohistochemistry (Figure S6C). The absence of nicotine-induced hypolocomotor effects observed in Gpr151-KO mice was recapitulated in shRNA-injected mice while re-expression of Gpr151 in the KO restored sensitivity to nicotine-induced hypolocomotion (Figure 2C), indicating that GPR151 acts in the MHb to regulate sensitivity to nicotine in adult mice.

Given that Gpr151-KO mice have an altered response to nicotine and that GPR151 is coexpressed with MOR and CB1R, we next evaluated the behavioral response of Gpr151-KO mice to morphine and the cannabinoid agonist arachidonyl-2'-chloroethylamide (ACEA). As expected, an acute injection of morphine resulted in progressive increase of locomotor activity in WT mice (Figure 2D) (Brase et al., 1977). In contrast Gpr151-KO mice were insensitive to the acute hyperlocomotor effect of morphine (Figure 2D). We monitored the development of sensitization to morphine over the course of 6 days of daily injections of morphine. WT mice developed locomotor sensitization while Gpr151-KO mice were resistant to sensitization (Figure 2E). Injection of the CB1R agonist ACEA induced hypoactivity as previously described (Almeida et al., 2014), which was indistinguishable between WT and Gpr151-KO mice (Figure S5E-F) consistent with studies showing that habenular CB1R does not influence locomotion (Soria-Gomez et al., 2015). However, since CB1R is critical for central regulation of food intake (Koch et al., 2015), we tested whether activation of CB1R induced a differential feeding response in Gpr151-KO mice. An injection of ACEA increased feeding in WT mice as described (Koch et al., 2015) while Gpr151-KO mice were insensitive to the hyperphagic effect of ACEA at $5 \mathrm{mg} / \mathrm{kg}$, and the hyperphagic effect at $10 \mathrm{mg} / \mathrm{kg}$ was significantly lower than WT mice (Figure 2F-G and Figure S5G-H). Similar to WT mice, 
Gpr151-KO mice injected in the $\mathrm{MHb}$ with the rescue virus demonstrated significantly increased food intake upon a $5 \mathrm{mg} / \mathrm{kg}$ injection of ACEA (Figure $2 \mathrm{H}$ ). Consecutive injections of ACEA over a 7-day period resulted in significant body weight gain in WT mice but not in Gpr151-KO mice (Figure 2I). Body weight of WT and Gpr151-KO mice on regular water and food was similar across age (Figure S5I). These data revealed an unsuspected role of GPR151 in feeding behavior induced by activation of CB1R. Prompted by this finding, and given the well-known anorectic effects of nicotine, we tested whether activation of nAChRs resulted in differential weight changes in Gpr151-KO mice. Mice were given drinking water containing nicotine and saccharin (to mask the bitter taste of nicotine) for 4 weeks. Control WT mice drinking only saccharin gained significantly more weight than WT mice treated with nicotine (Figure 2J). However, no differences in body weight were observed between Gpr151-KO mice treated either with saccharin or nicotine, indicating that Gpr151-KO mice are insensitive to the anorectic effects of nicotine.

These data suggest that GPR151 affects the sensitivity to the locomotor effects, feeding patterns and weight gain changes induced by nicotine, opioids and cannabinoids. The demonstration that Gpr151-KO mice do not differ from WT mice under baseline conditions, but respond differently to nicotine, morphine and ACEA, supports a critical role for GPR151 signalling in the $\mathrm{MHb}$ in controlling behavioral response to drugs of abuse across different classes. In addition, these results reveal an unsuspected role of the habenula in feeding regulation and weight control/energy balance in response to drugs of abuse.

\section{Reduced nicotine-induced suppression of feeding and increased self-administration of high nicotine doses in Gpr151-KO mice.}

To investigate whether GPR151 controls the reinforcing properties of addictive drugs, we examined WT and Gpr151-KO mice in a self-administration task. We focused our attention on 
nicotine because the MHb-IPN pathway is well-known to control consumption of this drug. Mice first underwent training to respond for food rewards in operant chambers where presses on the active lever resulted in the delivery of food pellets under a fixed-ratio 5 time-out 20 sec (FR5TO20) schedule of reinforcement. No differences were observed in the acquisition of lever pressing behavior (Figure S7). Based on the findings described above, suggesting that the $\mathrm{MHb}$ regulates the anorectic actions of nicotine and other drugs of abuse, we determined if nicotine (1mg/kg, s.c.) can reduce responding for food rewards in this task in WT and Gpr151-KO mice. In saline treated mice, the number of food pellets earned by WT mice (9.9 +/- 0.8) and Gpr151-KO mice (11.8 +/- 0.5) was comparable (Figure 3A). However, upon nicotine administration WT mice responded significantly less for food rewards than saline WT controls, consistent with the anorectic effect of nicotine on food intake. Similar to our findings with nicotine in the drinking water, this anorectic response was much less pronounced in Gpr151-KO mice (Figure 3A) reflecting a reduced sensitivity to nicotine-induced suppression of appetite.

Using the same paradigm but pairing the lever pressing to intravenous infusion of nicotine, we next investigated the role of GPR151 in nicotine reinforcement. As expected, WT mice responded for self-administered nicotine infusions according to a known inverted U-shaped dose-response curve (Fowler et al., 2011) (Figure 3B). Notably, Gpr151-KO mice selfadministered far more nicotine at a high nicotine dose than WT littermate mice (Figure 3B,C,E). This pattern of responding for nicotine, particularly at higher unit doses of the drug, is similar to that previously reported in a5 $\mathrm{nAChR}$ subunit $\mathrm{KO}$ mice and rats in which a5 subunits are selectively knocked down in Hb-IPN (Fowler et al., 2011), suggesting reduced sensitivity to the aversive effects of high doses of nicotine. Re-expression of GPR151 in the $\mathrm{MHb}$ of Gpr151-KO mice reduced self-administration of the $0.4 \mathrm{mg} / \mathrm{kg}$ nicotine dose to levels similar to WT mice (Figure 3D,E). Consistent with the locomotor behavioral tests (Figure 
$2 A, B, C)$, both the reduction in the anorectic effects of nicotine and the fact that Gpr151-KO mice self-administer more nicotine at a high doses suggests that the $\mathrm{KO}$ mice are resistant to the aversive and malaise-inducing effects of nicotine. Altogether, these results demonstrate that GPR151 is key in the inhibitory control exerted by the MHb in limiting drug and food intake.

\section{GPR151 is localized to axonal and presynaptic membranes and synaptic vesicles of habenular terminals.}

To understand the mechanism by which GPR151 modulates the sensitivity to nicotine, morphine and cannabinoids that act on their cognate habenular receptors, we next determined its precise subcellular localization. To this end, we employed immuno-electron microscopy (iEM) to visualize axonal projections and synaptic terminals in the rostral portion of the IPN (Figure 4A), which is most intensely labeled by GPR151 (Broms et al., 2015) and contains the highest concentration of cholinergic and glutamatergic axonal terminals arising from habenular neurons (Frahm et al., 2015). GPR151 immunoreactivity was observed in axon terminals of WT mice (Figure 4B and Figure S8A-B), but not in Gpr151-KO mice (Figure 4C and Figure S8C-D), confirming the specificity of the antibody. As expected from the characteristic architecture of the IPN, quantitative analysis of terminals containing an active zone (AZ) showed that most synaptic contacts labeled by GPR151 were axo-dendritic (50 out of 53), 2 were axo-axonic and 1 was axo-somatic (Figure S8E). To exclude the possibility that loss of GPR151 could have altered the morphology of IPN synapses we performed a comparative analysis of different synaptic markers in WT and Gpr151-KO terminals. No significant differences were observed in the area of the synaptic terminal, length of the $A Z$, diameter and density of SVs, and distance of SVs to the AZ (Figure 4D-H). 
The majority of GPR151 nanogold particles (73.63\%) were located at the presynaptic plasma membrane (Figure 4B and $4 \mathrm{~L}$ ), at the active zone (AZ) (5.19\%; Figure 4B, $4 \mathrm{I}$ and $4 \mathrm{~L}$ ) and in association with synaptic vesicles (SV) (9.66\% Figure $4 \mathrm{~J}$ and $4 \mathrm{~L})$ and dense core vesicles (DCV) (1.39\%, Figure 4K-L). SVs and DCVs labeled with GPR151 were mainly localized further than $200 \mathrm{~nm}$ from the AZ (Figure S8F-G). The distribution of GPR151 was similar in presynaptic terminals without an $A Z$ in the plane of the picture (Figure $S 8 B$ and $S 8 H$ ). GPR151 nanogold particles were also found along the habenular axonal projections, mostly at the membrane but also along the microtubules inside the axons (Figure $4 \mathrm{M}$ and Figure S9A-B). This is interesting in light of recent findings that $n A C h R s$ are also expressed along $\mathrm{Hb}$ axons in the fasciculus retroflexus, where they can be activated by nicotine (Passlick et al., 2018).

Next, we confirmed that GPR151 is at SVs by double postembedding iEM staining with the vesicular glutamate transporter 1 (VGLUT1) (Figure 4N-Q, Figure S9C-E). Out of 70 synaptic terminals analyzed, $87 \%(n=61)$ were labeled with both VGLUT1 and GPR151, while just $10 \%(n=7)$ were labeled with GPR151 only and $3 \%(n=2)$ were labeled with VGLUT1 only (Figure S9F). This indicates that around 90\% of GPR151 habenular terminals at the IPR are glutamatergic. GPR151 and VGLUT1 were found very close to each other in association with synaptic vesicles (Figure 4P) and along the microtubules within the axons being transported towards the terminals (Figure $4 \mathrm{Q}$ and S9G-H). In summary these experiments identify GPR151 as a novel and specific component of presynaptic habenular terminals in the IPN. The fact that Gpr151-KO mice have presynaptic terminals with normal morphology and SVs distribution argues against a role for GPR151 in synaptic scaffolding or cytoskeleton organization or in brain development. However, GPR151 localization at the synaptic and perisynaptic membrane and SVs suggests that GPR151 may modulate synaptic activity. 


\section{GPR151 contributes to synaptic fidelity, strength and plasticity.}

To explore the possibility that GPR151 modulates the synaptic activity of habenular terminals, we tested whether optogenetic activation of $\mathrm{MHb}$ terminals could reveal differences between WT and Gpr151-KO mice. Since the majority of GPR151 expressing neurons (70\%) are cholinergic MHb neurons (Figure S10), we crossed Gpr151-KO mice with transgenic mice expressing the light-gated cation channel channelrhodopsin-2 (ChR2) in cholinergic neurons (Chat-ChR2 mice) (Ren et al., 2011) and recorded IPN neurons from offspring mice (Figure 5A). eEPSCs were evoked by trains of $5 \mathrm{~ms}$ blue light pulses (Figure $5 B$ ) and the fidelity of transmission was calculated as the percentage of light pulses that resulted in detectable postsynaptic currents per second during 1 min (Figure $5 \mathrm{C}$ ). We observed increased fidelity rates in MHb-IPN synapses of Chat-ChR2xGpr151-KO mice compared to Chat-ChR2 mice (Figure 5C) and compared to Chat-ChR2xGpr151-KO mice injected with the rescue virus (AAV2/1-Gpr151) specifically in MHb (Figure 5D). However, the amplitude of the light evoked EPSCs was smaller in Chat-ChR2xGpr151-KO (Figure 5E). Next we used the Paired Pulse Ratio (PPR) to measure presynaptic release probability upon light stimulation. At basal conditions, the PPR was similar between genotypes (Figure 5F). However, nicotine application in Chat-ChR2 slices increases the probability of the initial presynaptic release of the readily releasable pool of vesicles and therefore reduces the PPR (Figure 5G, 5I). In contrast, the PPR of Chat-ChR2xGpr151-KO does not change upon nicotine application (Figure $5 \mathrm{H}-\mathrm{I}$ ). This is consistent with the fact that GPR151 regulates behavioral actions to nicotine, The increased synaptic fidelity, smaller EPSC and unchanged PPR upon nicotine administration in Chat-ChR2xGpr151-KO point to a critical role of GPR151 in maintaining the readily releasable pool (RRP) of synaptic vesicles in habenular terminals in the IPN. 


\section{GPR151 couples to the G-alpha inhibitory protein Gao1 and associates with} presynaptic components.

To understand the signaling mechanisms through which GPR151 controls habenular neurons we sought to identify the G-alpha protein subunit groups (Gas, Gai, Gaq/11, or Ga12/13,) it uses for signal transduction. To accomplish this, we collected brain samples from the IPN of WT and Gpr151-KO mice, immunoprecipitated (IP) the protein extracts with GPR151 antibodies and performed mass spectrometry analyses of IP samples (Figure 6A, Figure S11A and Table S4). As shown in the volcano plot (Figure 6B), GPR151 is the most enriched protein in the immunoprecipitated fraction in WT mice, and it is not present in Gpr151-KO extracts, confirming the efficacy and specificity of the IPs. In addition, we identified 17 proteins that co-immunoprecipitated with GPR151 (Table S4). Among these, we were especially interested in the interaction with the G-alpha inhibitory subunit Gnao1 (protein name Gao1), a heterotrimeric guanine nucleotide-binding G-proteins involved in intracellular signal transduction (Figure 6B). To confirm this interaction, we performed immunoprecipitations and Western blot analysis of IPN extracts from WT and KO mice. As shown in Figure 6C and 6D, GPR151 associates with Gao1 but not with the G-alpha stimulatory subunit (Gas). Consistently, the TRAP data shows that Gao1 is the most abundant of the inhibitory Gi/o proteins in cholinergic MHb neurons (Table S2). These results indicate that GPR151 couples to the G-alpha inhibitory pathway, which is known to inhibit adenylyl cyclase activity and therefore decrease intracellular cAMP levels.

Other proteins co-immunoprecipitated with GPR151 include synaptic vesicle proteins (Synaptic vesicle glycoprotein 2A (SV2A) and Voltage-Dependent Anion Channels (VDAC2)) (Morciano et al., 2009; Takamori et al., 2006) and proteins potentially involved in regulating the functional dynamics of the pre-synapse (including the sodium/potassium ATPase composed of AT1A2 and AT1A3 (catalytic a-subunits), AT1B1 and AT1B2 (structural $\beta$ - 
subunits)) the calcium ATPase (AT2A2 and AT2B3), and the $\mathrm{H}+$ transporting ATPase (AT5B) (Taruno et al., 2012; Zanni et al., 2012); (Figure 6B and Table S5, Figures S12, S13, S14 of GO analysis). Association of GPR151 with these proteins in mass spectrometry is consistent with immunoEM localization at the synaptic vesicle and with our electrophysiological findings demonstrating regulation of vesicular release.

\section{GPR151 regulates cAMP levels}

Given its interaction with Gao1, we next analyzed the possibility that GPR151 modulates cAMP levels. We conducted cAMP assays of IPN samples of WT and Gpr151-KO mice, as well as WT mice injected into the $\mathrm{MHb}$ with scramble shRNA or Gpr151-shRNA for downregulation (Figure 7A). Consistent with GPR151 coupling to Gao1, we observed that cAMP levels are higher in Gpr151-KO IPN homogenates (Figure 7B). In addition, we observed a strong trend of increased cAMP levels in Gpr151-downregulated IPN (Figure 7B). Given that cAMP is an important regulator of neurotransmission of $\mathrm{MHb}$ cholinergic synapses (Hu et al., 2012), we performed electrophysiological recordings in Chat-ChR2 and ChatChR2xGpr151-KO upon light stimulation as explained for Figure 5. As expected, addition of forskolin increased the amplitude of light evoked EPSCs in Chat-ChR2 mice (Figure 7C). However, the amplitude was not altered in Chat-ChR2xGpr151-KO upon forskolin application and remained smaller than WT levels (Figure 7C). This suggests that the absence of GPR151 compromises the coupling of cAMP signaling to the neurotransmitter release machinery in habenula neurons.

To further analyze the role of GPR151 in cAMP modulation and validate its interaction with Gao1, we generated two stable cell lines expressing GPR151 with an N-terminus 5HT3 signal peptide to enhance membrane expression (Wellerdieck et al., 1997; Wetzel et al., 
1999) plus a SNAP-tag to monitor surface expression (Ward et al., 2011) in HEK293 and $\mathrm{CHO}-\mathrm{K} 1$ cells. $\mathrm{CHO}-\mathrm{K} 1$ cells were used because they express high levels of Gao1. In contrast, HEK293 cells do not express Gao1, but express Gaz, which is a member of the same Gai/o family (Baycin-Hizal et al., 2012; Doi et al., 2016; Thakur et al., 2011). GPR151 expression at the membrane was validated by SNAP and GPR151 immunostaining and western blot (Figure 7D and S15). Next we measured cAMP levels upon forskolin stimulation using the LANCE cAMP assay. The $E_{50}$ of forskolin of GPR151-expressing HEK293 $(170.5 \mathrm{nM})$ or $\mathrm{CHO}-\mathrm{K} 1(167.1 \mathrm{nM})$ cells were significantly higher than their parental cells, HEK293 (25.8nM) and CHO-K1 (24.1nM) (Figure 7E-F), suggesting that GPR151 couples to Gai/o proteins and that its constitutive activity buffers the effect of forskolin.

Next we treated the cells with $250 \mathrm{ng} / \mathrm{ml}$ pertussis toxin (PT) overnight, which inactivates the G proteins by catalyzing the ADP-ribosylation of the asubunits of the heterotrimeric Gai/o protein family, with the exception the Gaz isoform (Fong et al., 1988; Matsuoka et al., 1988). PT had a strong effect on cAMP production stimulated by forskolin in GPR151-expressing $\mathrm{CHO}-\mathrm{K} 1$ cells (Figure $7 \mathrm{~F}$ ). The $\mathrm{EC}_{50}$ of forskolin decreased from $167.1 \mathrm{nM}$ to $71.82 \mathrm{nM}$ indicating that PT blocks a large portion of the inhibitory response mediated by Gao. The impact of PT in GPR15-expressing HEK293 cells is not as strong as in CHO-K1 cells (Figure 7E). The $\mathrm{EC}_{50}$ of forskolin in HEK293-GPR151 expressing cells decreased from $170.5 \mathrm{nM}$ to 146.3 nM upon PT treatment, most likely because HEK293 cells have Gaz, which is insensitive to PT.

Together these results provide evidence that GPR151 couples to Gao and decreases cAMP levels. In agreement with the lower cAMP levels observed in the IPN of WT mice compared to Gpr151-KO mice, GPR151 was observed to be constitutively active in both cell lines analyzed. This constitutive activity of GPR151 is actually not unexpected since it lacks the DRY motif which plays an essential role in class A-GPCR's function (Wess, 1998). Mutations 
or deletion of the DRY motif lead to the formation of active GPCR conformations (Rosenkilde et al., 2005; Wess, 1998). Moreover, GPCRs without the DRY motif have been shown to be both constitutively active and ligand-inducible; and that inserting the DRY motif decreases its constitutive activity (Rosenkilde et al., 2005). Our data suggest that GPR151 could be also functioning via both a constitutive and a ligand-inducible mechanism.

\section{DISCUSSION}

The conservation of the habenula across vertebrates and its emerging role in processing reward-related and aversive signals, including drug abuse responses, points to the need to further characterize this brain circuit and identify modulatory mechanisms that may provide common avenues for the development of new approaches towards the treatment of addiction and other disorders of the "reward" circuitry. Our previous studies showed the specific expression of the orphan receptor GPR151 in the habenula of rats, mice and zebrafish. Here, we establish conservation of the habenular-enrichment of this receptor in human brains, define its function in regulating habenular neurons, and reveal its critical role in regulating behavioral response to addictive drugs across different classes. More specifically, we demonstrate that GPR151 is present in presynaptic termini of rodent and human habenular neurons, and that it couples to Gao to regulate cAMP levels, synaptic fidelity and neurotransmission. We also show that neurons in the medial habenula co-express GPR151 with nAChRs, MOR and CB1R, and that loss of GPR151 results in alterations in behaviors associated with nicotine, opioid and cannabinoid use. Thus, our data identify the MHb/IPN projection as a common pathway controlling addictive-relevant behaviors in response to major classes of drugs of abuse, and they demonstrate that this important circuit is modulated by the orphan receptor GPR151. 
We found that GPR151 is selectively enriched in habenular presynaptic structures in the IPN. Several GPCRs, including dopamine D2, muscarinic M2 and M4, adrenergic, cannabinoid and opioid receptors have been localized at the presynapse where they inhibit evoked synaptic transmission (Betke et al., 2012). GPCRs directly at the active zone regulate fast transmission by local action of the dissociated G beta-gamma complex (GßY) on exocytosis, while GPCRs distant from the AZ modulate neurotransmission through second-messenger cascades (Betke et al., 2012). Following with this distinction, GPR151, which is mainly at the presynaptic plasma membrane (73\% gold particles), most likely regulates synaptic transmission through cAMP second messenger cascades rather than through Gßy. However, our mass spectrometry analysis revealed that GPR151 binds to Gao1 and G $\beta 1$ subunits, shown to localize to SVs and DCVs in neurons (Ahnert-Hilger et al., 1994; Takamori et al., 2006). The fact that GPR151 can be detected at the AZ, in SVs and in DCVs (17\% of immunoparticles) and that it immunoprecipitates with Gao1 and Gß1, suggests the presence of preassembled GPR151/G-protein signaling complexes in vesicles that can be transported to the plasma membrane upon neuronal stimulation. Interestingly, GPR151 can be activated by acidic conditions in vitro (Mashiko et al., 2019) suggesting the possibility that GPR151 is activated by acidic SVs which release protons during neurotransmission (Traynelis and Chesler, 2001). Acidification also occurs after neuronal injury and acidosis induces pain and hyperalgesia (Ortega-Ramirez et al., 2017). An increase of Gpr151 mRNA (but no GPR151 protein) has been detected after nerve ligation in dorsal root ganglia (Holmes et al., 2017) and spinal cord (Jiang et al., 2018). However, diminished allodynia in Gpr151-mutant mice after neuropathic pain was only found in one of the studies (Jiang et al., 2018), suggesting that further studies will be required to examine its role in pain.

Consistent with the EM analyses, GPR151 co-immunoprecipitates with proteins localized at presynaptic terminals and at SVs, again suggesting that GPR151 preassembles not only with 
G-alpha and beta proteins but also with SV components during its translocation to the plasma membrane. Importantly, GPR151 did not co-immunoprecipitate with CB1R and MOR, which are found presynaptically in habenular terminals, and we did not observe differences in CB1R and MOR protein expression in Gpr151-KO mice. These data exclude the possibility that the reduced sensitivity to opioids and cannabinoids in Gpr151-KO mice is caused by stoichiometric changes in the formation of heteromeric complexes of these receptors or their chaperoning to the membrane. This is in contrast to findings with antipsychotic drugs, which have been reported to bind glutamate mGluR2/ serotonin 2AR heterocomplexes to result in behavioral alterations in response to a variety of pharmacological compounds (Fribourg et al., 2011).

Our studies suggest that enhanced cAMP levels in habenular terminals of Gpr151-KO mice are responsible for the behavioral changes observed. CAMP reduces synaptic failure rates (Huang and Hsu, 2006) and facilitates neurotransmission by increasing release probability at central excitatory synapses (Sakaba and Neher, 2001). This has also been reported in habenular neurons where depletion of presynaptic cAMP levels suppresses neurotransmission ( $\mathrm{Hu}$ et al., 2012). Although enhanced synaptic fidelity improves the precision of information transfer through brain networks (Owen et al., 2013), in some cases, increased synaptic fidelity might be deleterious, for instance, when it is a consequence of chronic drug administration. Upregulation of the cAMP signaling pathway is a typical molecular adaptation in several brain regions following chronic morphine treatment (Bie et al., 2005), and activation of CB1R also modifies synaptic efficacy (Katona and Freund, 2008). MOR and CB1R, like GPR151, interact with the Gai/o class of adenylate cyclase inhibitory Ga proteins (Howlett and Fleming, 1984; Uhl et al., 1994). From these observations, it is reasonable to hypothesize that maintaining increased levels of cAMP at habenular terminals of Gpr151-KO blunts the response of these neurons to drugs of abuse. Thus, the picture 
emerging from our studies is that GPR151 constitutive activity in normal WT synapses maintains a low level of cAMP at habenular synapses that allows dynamic responses to fluctuation by drugs of abuse acting on habenular nicotinic, opioid and cannabinoid receptors. In Gpr151-KO mice, cAMP levels are very high and neither forskolin nor drugs of abuse can elicit changes of neurotransmitter release due to a ceiling effect. Since insensitivity to these drugs in Gpr151-KO mice leads to higher self-administration, the findings here suggest that future studies aimed at the identification of an agonist of GPR151 (that will further decrease the levels of CAMP at this synapse) could be useful to treat addiction. No studies in central synapses have evaluated whether nicotine increases cAMP levels, but it has been shown that nicotine acts presynaptically to augment the frequency of neurotransmitter release (Frahm et al., 2015). This suggests that in addition to changes in cAMP, other molecular mechanisms may determine release probability in habenular synapses, for instance through ATPases. Since GPR151 interacts with the sodium/potassium pump ATP1A3/B1 which contributes to precise and reliable transmission at glutamatergic synapses (Taruno et al., 2012), it is possible that GPR151 reduces synaptic strength during high-frequency stimulation by impairing ATP1A3/B1 trafficking or activity by regulating its phosphorylation (Poulsen et al., 2010). Further studies on this particular aspect would be key to understand how GPCRs contribute to ATPase function in synaptic fidelity.

Addictive drugs, although chemically different from one another, share common cellular and anatomical pathways. Two lines of investigation have led to this conclusion: 1) most of them activate the mesocorticolimbic dopaminergic (DA) reward circuitry, 2) each of them mimics or enhances the actions of one or more neurotransmitters in the brain that are involved in the control of the brain reward circuit (Koob and Volkow, 2010). Connections between the lateral habenula and the DA system have been established (either direct LHb-VTA, or via the RMTg-VTA-SNc) (Proulx et al., 2014), and between the MHb and VTA DA system via the 
IPN (Ables et al., 2017; Zhao-Shea et al., 2015). These observations suggest that drugs of abuse act at the MHb-IPN and mesocorticolimbic reward circuit to process both rewardrelated and aversive signals. Also we cannot exclude that Gpr151-KO behavioral effects may also involve the LHb since a subpopulation of LHb muscarinic M2 neurons is GPR151 positive. However consistent with our self-administration experiments, loss of GPR151 in habenula did not alter the rewarding effects of nicotine but abolished the inhibitory aversive effects of higher nicotine doses on brain reward. A balance between reward and aversion is also evident by the fact that not only tolerance, sensitization and self-administration responses are changed in Gpr151-KO mice, but also food intake by activation of CB1R and nAChRs. Animal studies and human imaging analyses have revealed that many psychoactive drugs act on the mesocorticolimbic dopamine system, a circuit that appears to be common to the rewarding effects of some drugs of abuse, as well as other reinforcing natural behaviors such as eating, thirst, gambling and sexual drive (Lammel et al., 2014; Lammel et al., 2012; Ostroumov and Dani, 2018). Feeding responses to drugs of abuse are also mediated by the mesolimbic DA circuit (Land et al., 2014; Nunes et al., 2013; Wise and McDevitt, 2018)and by hypothalamic POMC neurons (Koch et al., 2015; Mineur et al., 2011). Since neuroanatomical reciprocal connections exist between the IPN and hypothalamus (Groenewegen et al., 1986), it is possible that GPR151-mediated alterations of MHb-IPN neurotransmission impact feeding behavior by modulating postsynaptic responses of POMC neurons. It has been proposed that obesity and drug addiction arise from similar neuroadaptive responses (DiLeone et al., 2012; Kenny, 2011). Interestingly, UK biobank studies have recently associated GPR151 loss of function alleles to lower body mass index, warranting further studies on the role of Gpr151 in feeding. However we only detected differences on feeding behavior when mice are treated with the cannabinoid agonist ACEA (not with saline control animals) or with nicotine. Our results highlight the habenula as a common key brain area in the development of addiction to some drugs of abuse and provide 
evidence of a role of this ancient epithalamic nucleus in feeding regulation and weight control. Thus our findings raise the possibility that malfunction of the habenula may predispose individuals to drug consumption and consequent changes in food intake that may in turn reinforce the use of drugs.

Finally, our studies suggest GPR151 as a candidate target for therapies preventing drug abuse because: 1) it is exclusively localized in axonal projections of habenular neurons, decreasing therefore the likelihood of developing side effects due to alterations in other brain circuits or in the peripheral nervous system, 2) it modulates neurotransmission and by that affects sensitivity to nicotine, morphine and cannabinoid-induced behaviors, 3) it belongs to the highly druggable Class A family of GPCRs and can be screened with cAMP assays, and 4) its pattern of expression is conserved in the human habenula-IPN axis. We hope our work stimulates interest in this orphan GPCR since the identification of a GPR151 agonist would be a very valuable therapeutic target to reduce the enormous adverse impact of drug abuse on public health.

\section{EXPERIMENTAL PROCEDURES}

\section{Animals}

All procedures involving mice were approved by the Rockefeller University and Mount Sinai Institutional Animal Care and Use Committee and were in accordance with the National Institutes of Health guidelines. See Supplemental Experimental Procedures for further details.

\section{Co-immunoprecipitations, western blot and proteomic analysis}

Co-immunoprecipitations for mass spectrometry analysis were performed using $M$ 270 Epoxy Dynabeads (Invitrogen) with three different GPR151 antibodies (45 $\mu \mathrm{g}$ aprox) coupled to $15 \mathrm{mg}$ of beads per sample during $24 \mathrm{hr}$ at $37^{\circ} \mathrm{C}$ in $1.5 \mathrm{ml}$ volume. Co- 
immunoprecipitations for western blot analysis were done using $50 \mu \mathrm{l}$ of DynaBeads Protein G (Invitrogen) per sample and $6 \mu \mathrm{g}$ anti-GPR151 antibodies. See Supplemental Experimental Procedures for further details and proteomic analysis.

Immunohistochemistry of human and mouse brain samples and quantification analysis

Human brain tissues were obtained from the NICHD Brain and Tissue Bank from 5 donors, ranging between 22-52 years of age. Immunohistochemistry was performed in adult mice (812 weeks) as described in (Broms et al., 2015). See Supplemental Experimental Procedures for further details.

\section{Electron microscopy}

Pre-embedding and post-embedding nanogold labeling was performed as in (Frahm et al., 2015). See Supplemental Experimental Procedures for details.

\section{TRAP and RNA-SEQ}

Three biological replicates were used for TRAP analysis as described in (Mellen et al., 2012). Each replicate contained the habenulae from 5 CHAT-EGFP-L10a transgenic mice (males and females 8-12 weeks old). See Supplemental Experimental Procedures for details.

\section{Electrophysiological recordings}

IPN neurons were patch-clamped at $-70 \mathrm{mV}$. Presynaptic $\mathrm{MHb}$ fibers were excited for one continuous minute with a $473 \mathrm{~nm}$ blue light laser stimulation at $20 \mathrm{~Hz}$, and a pulse length of 5 ms. The fidelity of the MHb-IPN transmission was calculated for every second as the number of successful transmissions, divided by 20 in percent. Kinetics of successful transmissions were calculated using MiniAnalysis (Synaptosot). See Supplemental Experimental Procedures for details.

\section{Behavioral analysis}

All behavioral studies were conducted blind to the genotype of the tested mice and only male mice 8-16 weeks old were used. See Supplemental Experimental Procedures for details. 


\section{Intravenous nicotine self-administration procedure}

Mice were mildly food restricted to $85-90 \%$ of their free-feeding body weight and trained to press one of two levers in an operant chamber (Med-Associates Inc., St. Albans, VT) for food pellets under a FR5TO20 reinforcement schedule. When mice demonstrated stable responding (>25 pellets per session), they underwent jugular catheter implantation. Once stable responding was re-established after surgery, subjects were permitted to respond for intravenous nicotine infusions during $1 \mathrm{~h}$ daily sessions, 7 days per week, 3-5 days for each dose of nicotine in ascending order with saline last. In between each dose, subjects were permitted access to the training dose for at least 2 days or until their intake returned to baseline levels before being tested on the next dose. See Supplemental Experimental Procedures for more details.

\section{Statistical Analysis}

Statistical analyses were performed with GraphPad Prism 6.0. Unpaired two-tailed Student ttests, 2way ANOVA or Repeated Measures (RM) 2way ANOVA were used for analyzing most of the data as indicated in figure legends. See Table S3 for details of statistical analysis. Results are presented as means \pm S.E.M.

\section{AUTHOR CONTRIBUTIONS}

Study concept and design: PJK and II-T Acquisition of data: BA-F, AG, JLA, MD. Analysis and interpretation of data: BA-F, AG, JLA, PJK, and II-T. Writing of the manuscript: BA-F, PJK and II-T.

\section{ACKNOWLEDGMENTS}

We thank Kunihiro Uryu and Nadine Soplop (Electron Microscopy Resource Center, Rockefeller University) for EM expertise. We thank Milica Tešić Mark and Henrik Molina (Proteomics Resource Center, The Rockefeller University) for mass spectrometry analysis, 
which acknowledges funding from the Leona M. and Harry B. Helmsley Charitable Trust. Human tissue was obtained from the NICHD Brain and Tissue Bank for Developmental Disorders at the University of Maryland, Baltimore, MD. We thank Awni Mousa for analysis of RNA-Seq data, Sylvia M. Lipford, Cuidong Wang and Juncheng Li for technical assistance and Kun Li and Nathaniel Heintz for critical discussions. A.G received a DFG fellowship (GO 2334/1-1). This work was supported by the Leona M. and Harry B. Helmsley Charitable Trust and Sohn Conferences Foundation for mass spectrometer instrumentation (The Rockefeller University Proteomics Resource Center), the Leon Black Family Foundation (IIT), NIDA (1P30 DA035756-01) (II-T), DA020686 (PJK)), and NIDA UG3 DA048385(PJK and IIT).

\section{REFERENCES}

Ables, J.L., Gorlich, A., Antolin-Fontes, B., Wang, C., Lipford, S.M., Riad, M.H., Ren, J., Hu, F., Luo, M., Kenny, P.J., et al. (2017). Retrograde inhibition by a specific subset of interpeduncular alpha5 nicotinic neurons regulates nicotine preference. Proceedings of the National Academy of Sciences of the United States of America 114, $13012-13017$.

Agetsuma, M., Aizawa, H., Aoki, T., Nakayama, R., Takahoko, M., Goto, M., Sassa, T., Amo, R., Shiraki, T., Kawakami, K., et al. (2010). The habenula is crucial for experience-dependent modification of fear responses in zebrafish. Nat Neurosci 13, 1354-1356.

Ahnert-Hilger, G., Schafer, T., Spicher, K., Grund, C., Schultz, G., and Wiedenmann, B. (1994). Detection of G-protein heterotrimers on large dense core and small synaptic vesicles of neuroendocrine and neuronal cells. Eur J Cell Biol 65, 26-38.

Almeida, V., Peres, F.F., Levin, R., Suiama, M.A., Calzavara, M.B., Zuardi, A.W., Hallak, J.E., Crippa, J.A., and Abilio, V.C. (2014). Effects of cannabinoid and vanilloid drugs on positive and negative-like symptoms on an animal model of schizophrenia: the SHR strain. Schizophr Res 153, 150-159.

Antolin-Fontes, B., Ables, J.L., Gorlich, A., and Ibanez-Tallon, I. (2015). The habenulo-interpeduncular pathway in nicotine aversion and withdrawal. Neuropharmacology.

Baycin-Hizal, D., Tabb, D.L., Chaerkady, R., Chen, L., Lewis, N.E., Nagarajan, H., Sarkaria, V., Kumar, A., Wolozny, D., Colao, J., et al. (2012). Proteomic analysis of Chinese hamster ovary cells. J Proteome Res 11, 5265-5276. 
Betke, K.M., Wells, C.A., and Hamm, H.E. (2012). GPCR mediated regulation of synaptic transmission. Progress in neurobiology 96, 304-321.

Bie, B., Peng, Y., Zhang, Y., and Pan, Z.Z. (2005). cAMP-mediated mechanisms for pain sensitization during opioid withdrawal. The Journal of neuroscience : the official journal of the Society for Neuroscience 25, 3824-3832.

Boulos, L.J., Ben Hamida, S., Bailly, J., Maitra, M., Ehrlich, A.T., Gaveriaux-Ruff, C., Darcq, E., and Kieffer, B.L. (2019). Mu opioid receptors in the medial habenula contribute to naloxone aversion. Neuropsychopharmacology : official publication of the American College of Neuropsychopharmacology.

Brase, D.A., Loh, H.H., and Way, E.L. (1977). Comparison of the effects of morphine on locomotor activity, analgesia and primary and protracted physical dependence in six mouse strains. The Journal of pharmacology and experimental therapeutics 201, 368-374.

Broms, J., Antolin-Fontes, B., Tingstrom, A., and Ibanez-Tallon, I. (2015). Conserved expression of the GPR151 receptor in habenular axonal projections of vertebrates. The Journal of comparative neurology 523, 359-380.

Changeux, J.P. (2010). Nicotine addiction and nicotinic receptors: lessons from genetically modified mice. Nature reviews Neuroscience 11, 389-401.

Clarke, P.B., and Kumar, R. (1983). The effects of nicotine on locomotor activity in non-tolerant and tolerant rats. British journal of pharmacology 78, 329-337.

DiLeone, R.J., Taylor, J.R., and Picciotto, M.R. (2012). The drive to eat: comparisons and distinctions between mechanisms of food reward and drug addiction. Nat Neurosci 15, 1330-1335.

Doi, M., Murai, I., Kunisue, S., Setsu, G., Uchio, N., Tanaka, R., Kobayashi, S., Shimatani, H., Hayashi, H., Chao, H.W., et al. (2016). Gpr176 is a Gz-linked orphan G-protein-coupled receptor that sets the pace of circadian behaviour. Nat Commun 7, 10583.

Doyle, J.P., Dougherty, J.D., Heiman, M., Schmidt, E.F., Stevens, T.R., Ma, G., Bupp, S., Shrestha, P., Shah, R.D., Doughty, M.L., et al. (2008). Application of a translational profiling approach for the comparative analysis of CNS cell types. Cell 135, 749-762.

Ellison, G. (2002). Neural degeneration following chronic stimulant abuse reveals a weak link in brain, fasciculus retroflexus, implying the loss of forebrain control circuitry. Eur Neuropsychopharmacol 12, 287-297.

Fong, H.K., Yoshimoto, K.K., Eversole-Cire, P., and Simon, M.I. (1988). Identification of a GTP-binding protein alpha subunit that lacks an apparent ADP-ribosylation site for pertussis toxin. Proceedings of the National Academy of Sciences of the United States of America 85, 3066-3070.

Fowler, C.D., Lu, Q., Johnson, P.M., Marks, M.J., and Kenny, P.J. (2011). Habenular alpha5 nicotinic receptor subunit signalling controls nicotine intake. Nature 471 , 597-601.

Frahm, S., Antolin-Fontes, B., Gorlich, A., Zander, J.F., Ahnert-Hilger, G., and Ibanez-Tallon, I. (2015). An essential role of acetylcholine-glutamate synergy at habenular synapses in nicotine dependence. eLife 4.

Frahm, S., Slimak, M.A., Ferrarese, L., Santos-Torres, J., Antolin-Fontes, B., Auer, S., Filkin, S., Pons, S., Fontaine, J.F., Tsetlin, V., et al. (2011). Aversion to nicotine 
is regulated by the balanced activity of beta4 and alpha5 nicotinic receptor subunits in the medial habenula. Neuron 70, 522-535.

Fribourg, M., Moreno, J.L., Holloway, T., Provasi, D., Baki, L., Mahajan, R., Park, G., Adney, S.K., Hatcher, C., Eltit, J.M., et al. (2011). Decoding the signaling of a GPCR heteromeric complex reveals a unifying mechanism of action of antipsychotic drugs. Cell 147, 1011-1023.

Gardon, O., Faget, L., Chu Sin Chung, P., Matifas, A., Massotte, D., and Kieffer, B.L. (2014). Expression of $\mathrm{mu}$ opioid receptor in dorsal diencephalic conduction system: new insights for the medial habenula. Neuroscience 277, 595-609.

Gorlich, A., Antolin-Fontes, B., Ables, J.L., Frahm, S., Slimak, M.A., Dougherty, J.D., and Ibanez-Tallon, I. (2013). Reexposure to nicotine during withdrawal increases the pacemaking activity of cholinergic habenular neurons. Proceedings of the National Academy of Sciences of the United States of America 110, 17077-17082.

Groenewegen, H.J., Ahlenius, S., Haber, S.N., Kowall, N.W., and Nauta, W.J. (1986). Cytoarchitecture, fiber connections, and some histochemical aspects of the interpeduncular nucleus in the rat. The Journal of comparative neurology 249, 65102.

Haines, D.E. (2004). Neuroanatomy: an atlas of structured, sections and systems. Lippincott Williams \& Wilkins, sixth edition.

Holmes, F.E., Kerr, N., Chen, Y.J., Vanderplank, P., McArdle, C.A., and Wynick, D. (2017). Targeted disruption of the orphan receptor Gpr151 does not alter painrelated behaviour despite a strong induction in dorsal root ganglion expression in a model of neuropathic pain. Mol Cell Neurosci 78, 35-40.

Howlett, A.C., and Fleming, R.M. (1984). Cannabinoid inhibition of adenylate cyclase. Pharmacology of the response in neuroblastoma cell membranes. Mol Pharmacol 26, 532-538.

Hu, F., Ren, J., Zhang, J.E., Zhong, W., and Luo, M. (2012). Natriuretic peptides block synaptic transmission by activating phosphodiesterase $2 \mathrm{~A}$ and reducing presynaptic PKA activity. Proceedings of the National Academy of Sciences of the United States of America 109, 17681-17686.

Huang, C.C., and Hsu, K.S. (2006). Presynaptic mechanism underlying cAMPinduced synaptic potentiation in medial prefrontal cortex pyramidal neurons. Mol Pharmacol 69, 846-856.

Jiang, B.C., Zhang, W.W., Yang, T., Guo, C.Y., Cao, D.L., Zhang, Z.J., and Gao, Y.J. (2018). Demethylation of G-Protein-Coupled Receptor 151 Promoter Facilitates the Binding of Kruppel-Like Factor 5 and Enhances Neuropathic Pain after Nerve Injury in Mice. The Journal of neuroscience : the official journal of the Society for Neuroscience 38, 10535-10551.

Katona, I., and Freund, T.F. (2008). Endocannabinoid signaling as a synaptic circuit breaker in neurological disease. Nat Med 14, 923-930.

Kenny, P.J. (2011). Common cellular and molecular mechanisms in obesity and drug addiction. Nature reviews Neuroscience 12, 638-651.

Kobayashi, Y., Sano, Y., Vannoni, E., Goto, H., Suzuki, H., Oba, A., Kawasaki, H., Kanba, S., Lipp, H.P., Murphy, N.P., et al. (2013). Genetic dissection of medial habenula-interpeduncular nucleus pathway function in mice. Front Behav Neurosci $7,17$. 
Koch, M., Varela, L., Kim, J.G., Kim, J.D., Hernandez-Nuno, F., Simonds, S.E., Castorena, C.M., Vianna, C.R., Elmquist, J.K., Morozov, Y.M., et al. (2015). Hypothalamic POMC neurons promote cannabinoid-induced feeding. Nature 519, 45-50.

Koob, G.F., and Volkow, N.D. (2010). Neurocircuitry of addiction. Neuropsychopharmacology : official publication of the American College of Neuropsychopharmacology 35, 217-238.

Koob, G.F., and Volkow, N.D. (2016). Neurobiology of addiction: a neurocircuitry analysis. Lancet Psychiatry 3, 760-773.

Lammel, S., Lim, B.K., and Malenka, R.C. (2014). Reward and aversion in a heterogeneous midbrain dopamine system. Neuropharmacology 76 Pt B, 351-359.

Lammel, S., Lim, B.K., Ran, C., Huang, K.W., Betley, M.J., Tye, K.M., Deisseroth, K., and Malenka, R.C. (2012). Input-specific control of reward and aversion in the ventral tegmental area. Nature 491, 212-217.

Land, B.B., Narayanan, N.S., Liu, R.J., Gianessi, C.A., Brayton, C.E., Grimaldi, D.M., Sarhan, M., Guarnieri, D.J., Deisseroth, K., Aghajanian, G.K., et al. (2014). Medial prefrontal D1 dopamine neurons control food intake. Nat Neurosci 17, 248-253.

Mashiko, M., Kurosawa, A., Tani, Y., Tsuji, T., and Takeda, S. (2019). GPR31 and GPR151 are activated under acidic conditions. J Biochem.

Mathis, V., and Kenny, P.J. (2018). From controlled to compulsive drug-taking: The role of the habenula in addiction. Neurosci Biobehav Rev.

Matsuoka, M., Itoh, H., Kozasa, T., and Kaziro, Y. (1988). Sequence analysis of cDNA and genomic DNA for a putative pertussis toxin-insensitive guanine nucleotide-binding regulatory protein alpha subunit. Proceedings of the National Academy of Sciences of the United States of America 85, 5384-5388.

McLaughlin, I., Dani, J.A., and De Biasi, M. (2017). The medial habenula and interpeduncular nucleus circuitry is critical in addiction, anxiety, and mood regulation. Journal of neurochemistry 142 Suppl 2, 130-143.

Mellen, M., Ayata, P., Dewell, S., Kriaucionis, S., and Heintz, N. (2012). MeCP2 binds to $5 \mathrm{hmC}$ enriched within active genes and accessible chromatin in the nervous system. Cell 151, 1417-1430.

Mineur, Y.S., Abizaid, A., Rao, Y., Salas, R., DiLeone, R.J., Gundisch, D., Diano, S., De Biasi, M., Horvath, T.L., Gao, X.B., et al. (2011). Nicotine decreases food intake through activation of POMC neurons. Science 332, 1330-1332.

Morciano, M., Beckhaus, T., Karas, M., Zimmermann, H., and Volknandt, W. (2009). The proteome of the presynaptic active zone: from docked synaptic vesicles to adhesion molecules and maxi-channels. Journal of neurochemistry 108, 662-675.

Neugebauer, N.M., Einstein, E.B., Lopez, M.B., McClure-Begley, T.D., Mineur, Y.S., and Picciotto, M.R. (2013). Morphine dependence and withdrawal induced changes in cholinergic signaling. Pharmacology, biochemistry, and behavior 109, 77-83.

Nunes, E.J., Randall, P.A., Podurgiel, S., Correa, M., and Salamone, J.D. (2013). Nucleus accumbens neurotransmission and effort-related choice behavior in food motivation: effects of drugs acting on dopamine, adenosine, and muscarinic acetylcholine receptors. Neurosci Biobehav Rev 37, 2015-2025. 
Nutt, D.J., Lingford-Hughes, A., Erritzoe, D., and Stokes, P.R. (2015). The dopamine theory of addiction: 40 years of highs and lows. Nature reviews Neuroscience 16, 305-312.

Ortega-Ramirez, A., Vega, R., and Soto, E. (2017). Acid-Sensing lon Channels as Potential Therapeutic Targets in Neurodegeneration and Neuroinflammation. Mediators Inflamm 2017, 3728096.

Ostroumov, A., and Dani, J.A. (2018). Inhibitory Plasticity of Mesocorticolimbic Circuits in Addiction and Mental Illness. Trends in neurosciences 41, 898-910.

Owen, S.F., Tuncdemir, S.N., Bader, P.L., Tirko, N.N., Fishell, G., and Tsien, R.W. (2013). Oxytocin enhances hippocampal spike transmission by modulating fastspiking interneurons. Nature 500, 458-462.

Passlick, S., Thapaliya, E.R., Chen, Z., Richers, M.T., and Ellis-Davies, G.C.R. (2018). Optical probing of acetylcholine receptors on neurons in the medial habenula with a novel caged nicotine drug analogue. The Journal of physiology 596, 5307-5318.

Poulsen, H., Morth, P., Egebjerg, J., and Nissen, P. (2010). Phosphorylation of the $\mathrm{Na}+, \mathrm{K}+-\mathrm{ATP}$ ase and the $\mathrm{H}_{+}, \mathrm{K}+-$ ATPase. FEBS Lett 584, 2589-2595.

Proulx, C.D., Hikosaka, O., and Malinow, R. (2014). Reward processing by the lateral habenula in normal and depressive behaviors. Nat Neurosci 17, 1146-1152.

Ren, J., Qin, C., Hu, F., Tan, J., Qiu, L., Zhao, S., Feng, G., and Luo, M. (2011). Habenula "cholinergic" neurons co-release glutamate and acetylcholine and activate postsynaptic neurons via distinct transmission modes. Neuron 69, 445452.

Rosenkilde, M.M., Kledal, T.N., and Schwartz, T.W. (2005). High constitutive activity of a virus-encoded seven transmembrane receptor in the absence of the conserved DRY motif (Asp-Arg-Tyr) in transmembrane helix 3. Mol Pharmacol 68, 11-19.

Sakaba, T., and Neher, E. (2001). Preferential potentiation of fast-releasing synaptic vesicles by cAMP at the calyx of Held. Proceedings of the National Academy of Sciences of the United States of America 98, 331-336.

Salas, R., Sturm, R., Boulter, J., and De Biasi, M. (2009). Nicotinic receptors in the habenulo-interpeduncular system are necessary for nicotine withdrawal in mice. The Journal of neuroscience : the official journal of the Society for Neuroscience 29, 3014-3018.

Shih, P.Y., Engle, S.E., Oh, G., Deshpande, P., Puskar, N.L., Lester, H.A., and Drenan, R.M. (2014). Differential expression and function of nicotinic acetylcholine receptors in subdivisions of medial habenula. The Journal of neuroscience : the official journal of the Society for Neuroscience 34, 9789-9802.

Simonnet, A., Zamberletti, E., Cador, M., Rubino, T., and Caille, S. (2017). Chronic FAAH inhibition during nicotine abstinence alters habenular CB1 receptor activity and precipitates depressive-like behaviors. Neuropharmacology 113, 252-259.

Soria-Gomez, E., Busquets-Garcia, A., Hu, F., Mehidi, A., Cannich, A., Roux, L., Louit, I., Alonso, L., Wiesner, T., Georges, F., et al. (2015). Habenular CB1 Receptors Control the Expression of Aversive Memories. Neuron 88, 306-313. 
Takamori, S., Holt, M., Stenius, K., Lemke, E.A., Gronborg, M., Riedel, D., Urlaub, H., Schenck, S., Brugger, B., Ringler, P., et al. (2006). Molecular anatomy of a trafficking organelle. Cell 127, 831-846.

Taruno, A., Ohmori, H., and Kuba, H. (2012). Inhibition of presynaptic $\mathrm{Na}(+) / \mathrm{K}(+)$ ATPase reduces readily releasable pool size at the avian end-bulb of Held synapse. Neurosci Res 72, 117-128.

Thakur, S.S., Geiger, T., Chatterjee, B., Bandilla, P., Frohlich, F., Cox, J., and Mann, M. (2011). Deep and highly sensitive proteome coverage by LC-MS/MS without prefractionation. Mol Cell Proteomics 10, M110 003699.

Traynelis, S.F., and Chesler, M. (2001). Proton release as a modulator of presynaptic function. Neuron 32, 960-962.

Uhl, G.R., Childers, S., and Pasternak, G. (1994). An opiate-receptor gene family reunion. Trends in neurosciences 17, 89-93.

Velasquez, K.M., Molfese, D.L., and Salas, R. (2014). The role of the habenula in drug addiction. Frontiers in human neuroscience 8, 174.

Ward, R.J., Pediani, J.D., and Milligan, G. (2011). Ligand-induced internalization of the orexin $\mathrm{OX}(1)$ and cannabinoid $\mathrm{CB}(1)$ receptors assessed via N-terminal SNAP and CLIP-tagging. British journal of pharmacology 162, 1439-1452.

Wellerdieck, C., Oles, M., Pott, L., Korsching, S., Gisselmann, G., and Hatt, H. (1997). Functional expression of odorant receptors of the zebrafish Danio rerio and of the nematode C. elegans in HEK293 cells. Chem Senses 22, 467-476.

Wess, J. (1998). Molecular basis of receptor/G-protein-coupling selectivity. Pharmacol Ther 80, 231-264.

Wetzel, C.H., Oles, M., Wellerdieck, C., Kuczkowiak, M., Gisselmann, G., and Hatt, H. (1999). Specificity and sensitivity of a human olfactory receptor functionally expressed in human embryonic kidney 293 cells and Xenopus Laevis oocytes. The Journal of neuroscience : the official journal of the Society for Neuroscience 19, 7426-7433.

WHO https://www. who.int/substance abuse/en/. Accessed 06-24-19.

Wise, R.A., and McDevitt, R.A. (2018). Drive and Reinforcement Circuitry in the Brain: Origins, Neurotransmitters, and Projection Fields. Neuropsychopharmacology : official publication of the American College of Neuropsychopharmacology 43, 680-689.

Zanni, G., Cali, T., Kalscheuer, V.M., Ottolini, D., Barresi, S., Lebrun, N., MontecchiPalazzi, L., Hu, H., Chelly, J., Bertini, E., et al. (2012). Mutation of plasma membrane Ca2+ ATPase isoform 3 in a family with X-linked congenital cerebellar ataxia impairs $\mathrm{Ca2}+$ homeostasis. Proceedings of the National Academy of Sciences of the United States of America 109, 14514-14519.

Zhao-Shea, R., DeGroot, S.R., Liu, L., Vallaster, M., Pang, X., Su, Q., Gao, G., Rando, O.J., Martin, G.E., George, O., et al. (2015). Increased CRF signalling in a ventral tegmental area-interpeduncular nucleus-medial habenula circuit induces anxiety during nicotine withdrawal. Nat Commun 6, 6770.

\section{FIGURE LEGENDS}




\section{Figure 1: The habenula-IPN pathway is enriched in GPR151, nicotinic, opioid and} cannabinoid receptors

(A-B) Sagittal and coronal mouse brain scheme indicating the habenula (medial (MHb) and lateral (LHb), fasciculus retroflexus (FR) and interpeduncular nucleus (IPN).

(C-D) GPR151 is expressed in the axonal fibers originating mostly in MHb and passing through the LHb to form the FR (C; Scale bar: $100 \mu \mathrm{m})$; and along the FR and at axonal terminals in the IPN (D; Scale bar: $500 \mu \mathrm{m})$.

(E-F) Sagittal and coronal human brain sections indicating the habenula and IPN. Dotted lines show the plane of section in $\mathrm{G}, \mathrm{J}$, and M. Image modified from (Haines, 2004). Diagram highlighting the MHb, LHb and FR (adapted from Allen Brain Human Atlas).

(G-I) Coronal and horizontal sections of human brain samples showing GPR151 immunostaining in the MHb, LHb and beginning of the FR (G), along the FR (J) and IPN (M). Punctuate pattern of GPR151 was observed along the axons and in the IPN (arrows) on high magnification pictures of the square areas indicated. Scale bar: $1 \mathrm{~mm}$ in $\mathrm{G}, 50) \mu \mathrm{m}$ in $\mathrm{J}$ and $\mathrm{M}$, $50 \mu \mathrm{m}$ in $\mathrm{H}, \mathrm{K}$ and $\mathrm{N}$ and $20 \mu \mathrm{m}$ in $\mathrm{I}, \mathrm{L}$ and $\mathrm{O}$.

(P) Western blot analysis of untransfected and GPR151-HA transfected HEK293 cells; immunoprecipitated IPN samples from WT, Gpr151-KO mice, human IPN and human midbrain. Two bands (46 and $53 \mathrm{kDa}$ ) correspond to GPR151.

(Q) TRAP data collected from MHb neurons of Chat-EGFP-L10a mice crossed to WT and Gpr151-KO show high and similar expression levels (z-score transformed normalized counts) of Chat, opioid (Oprm1) cannabinoid (Cnr1) and nicotinic (Chrnb2,Chrna3 Chrnb4, Chrna4, Chrnb3) and Slc17a7 (VGLUT1) translated mRNAs in IP samples compared to Input samples. Only Gpr151 shows differential expression between WT and Gpr151-KO IP samples ( $\mathrm{n}=6 \mathrm{WT}, 6 \mathrm{KO})$.

(R) Scheme of the Hb-IPN subnuclei (IPR: rostral, IPL: lateral, IPI: intermediate, IPC: central). 
(S-V) Double immunostaining of GPR151 and nAChRa3-GFP (S), $\mu$-opioid receptor (MOR)

(T) and cannabinoid receptor 1 (CB1R) (V) showing high coexpression in the IPR where Manders' co-localization index (M1) and significance of correlation (P) were measured (indicated square area). Scale bar: $100 \mu \mathrm{m}$ for low magnification pictures, $20 \mu \mathrm{m}$ for high magnification pictures.

\section{Figure 2. Gpr151-KO mice show blunted behavioral responses to nicotine, morphine}

\section{and cannabinoids.}

(A) Hypolocomotion induced by acute injection of nicotine $(0.65 \square \mathrm{mg} / \mathrm{kg}$, i.p. $)$, measured as number of beam breaks in 5 min intervals, is significantly diminished in Gpr151-KO mice ( $\mathrm{n}=7$ per genotype; RM 2-way ANOVA, Bonferroni multiple comparisons ${ }^{*} p<0.05$, ${ }^{* *} p<0.01$ for genotype; Tukey's multiple comparisons test \#\#\#p<0.001 for time in both groups). Basal locomotor activity $(\min 0)$ is similar between groups.

(B) Consecutive daily injections of nicotine induce tolerance to the hypolocomotor effects of nicotine in WT but not in Gpr151-KO mice ( $\mathrm{n}=7$ per genotype, RM 2-way ANOVA, Bonferroni multiple comparisons $\# p<0.05$ for genotype; Tukey's multiple comparisons test ${ }^{* *} p<0.01$, ${ }^{* * *} p<0.001$ for time).

(C) Hypolocomotion induced by nicotine is observed at the higher 2 doses of nicotine $(0.65$ and $1.5 \mathrm{mg} / \mathrm{kg}$ ) in WT mice, WT mice injected with AAV2.1-scramble-shRNA (scramble) and in Gpr151-KO mice injected with AAV2.1-CAG-Gpr151 (rescue) in the MHb, but not in Gpr151-KO mice and in WT mice injected with AAV2.1-shRNA against Gpr151 (shRNA) in the $\mathrm{MHb}$. No differences were observed between genotypes upon saline injection or a low dose of nicotine $(0.35 \mathrm{mg} / \mathrm{kg})$. ( $\mathrm{n}=7-8$ per genotype, RM 2-way ANOVA, Tukey's multiple comparison test ${ }^{*} p<0.05,{ }^{* *} p<0.01,{ }^{* * *} p<0.001$ for genotype; $\# \# p<0.01, \quad \# \# \# p<0.001$ compared to $0.35 \mathrm{mg} / \mathrm{kg}$ nicotine within the same genotype). 
(D) Acute injection of morphine (10 $\square \mathrm{mg} / \mathrm{kg}$, s.c.) induces hyperlocomotion in WT mice, but not in Gpr151-KO mice ( $\mathrm{n}=7-8$ per genotype; RM 2-way ANOVA, Bonferroni multiple comparisons ${ }^{*} \mathrm{p}<0.05,{ }^{* *} \mathrm{p}<0.01,{ }^{* * *} \mathrm{p}<0.001$ for genotype; Tukey's multiple comparisons test $\# \# p<0.01$ for time).

(E) Consecutive daily injections of morphine induce sensitization to hyperlocomotor activity in WT mice but not in Gpr151-KO mice. (n=7-8 per genotype; RM 2-way ANOVA, Tukey's multiple comparisons test $\left.{ }^{* * *} \mathrm{p}<0.001\right)$.

(F) Acute injection of the cannabinoid agonist ACEA (5mg/kg, i.p.) increases food intake in WT mice but not in Gpr151-KO mice (n=8-7 per genotype; RM 2-way ANOVA, Bonferroni's multiple comparisons test, ${ }^{* *} \mathrm{p}<0.01$ in WT mice when comparing saline injection to ACEA injection, \#p<0.05 when comparing WT to Gpr151-KO injected with ACEA).

(G) Hyperphagia induced by ACEA is observed at the higher 2 doses of ACEA (5 and 10 $\mathrm{mg} / \mathrm{kg}$ ) in WT mice and only at the high dose in Gpr151-KO mice ( $\mathrm{n}=10-8$ per genotype; RM 2-way ANOVA, Bonferroni's multiple comparisons test for genotype, ${ }^{*} p<0.05,{ }^{* *} p<0.01$; Tukey's multiple comparisons test for ACEA concentration compared to saline, $\# \#$ \# $<0.0001)$.

(H) Gpr151-KO mice injected with AAV2.1-CAG-Gpr151 in the MHb (rescue virus) but not with control virus show increased food intake after injection of ACEA $(5 \mathrm{mg} / \mathrm{kg})$ ( $\mathrm{n}=9$ per genotype; RM 2-way ANOVA, Bonferroni's multiple comparisons test for genotype, * $\mathrm{p}<0.05$; Tukey's multiple comparisons test for ACEA concentration compared to saline, \#\# $p<0.01$ ).

(I) Daily injections of ACEA (5mg/kg, i.p.) for 7 days increase the body weight of WT mice but not of Gpr151-KO mice ( $\mathrm{n}=8-7$ per genotype; RM 2-way ANOVA, Bonferroni's multiple comparisons test, $\left.{ }^{* * *} \mathrm{p}<0.001\right)$.

(J) Mice consuming $162,5 \mu \mathrm{g} / \mathrm{ml}$ nicotine $+2 \%$ saccharin in the drinking water for 28 days show less increase of body weight with respect to mice treated with only $2 \%$ saccharin. No effect of nicotine on body weight was observed between Gpr151-KO mice treated with 
saccharin or nicotine ( $\mathrm{n}=11-26$ per group; RM 2-way ANOVA, Sidak's multiple comparisons test, * $\mathrm{p}<0.05$ when comparing WT saccharin to WT nicotine on day 28$)$.

Data are represented as mean \pm SEM. See Table S3 for details of statistical analysis.

\section{Figure 3. Gpr151-KO mice self-administer more nicotine at a high dose.}

(A) Average number of food rewards after injection of saline or nicotine $(1 \mathrm{mg} / \mathrm{kg}$, s.c.). Gpr151-KO mice are resistant to the anorectic effect of nicotine ( $\mathrm{n}=10$ per group, RM 2-way ANOVA, Bonferroni's multiple comparisons test ${ }^{* *} \mathrm{p}<0.01$ compared to WT).

(B) Gpr151-KO mice earned significantly more nicotine infusions at the higher nicotine dose (0.4 mg/kg) ( $\mathrm{n}=4-8, \mathrm{RM}$ 2-way ANOVA, Bonferroni's multiple comparisons test ${ }^{* *} \mathrm{p}<0.01$ compared to WT).

(C) Total amount of nicotine self-administered at each dose ( $n=4-8, R M 2$-way ANOVA, Bonferroni's multiple comparisons test ${ }^{* *} \mathrm{p}<0.001$ compared to WT). Gpr151-KO mice selfadminister a significantly higher amount of nicotine at the highest $0.4 \mathrm{mg} / \mathrm{kg} / \mathrm{session}$ dose.

(D) Re-expression of GPR151 in the Mhb and IPN terminals of Gpr151-KO mice injected with AAV2.1-Gpr151 visualized by GPR151 immunoreactivity and comparison with Gpr151-KO mice injected with AAV2/1- EGFP control virus. Scale bar: 500 um.

(E) At the highest nicotine dose $(0.4 \mathrm{mg} / \mathrm{kg} / \mathrm{session})$, Gpr151-KO mice injected with the AAV2.1-Gpr151 rescue virus significantly decrease the amount of self-administered nicotine to similar levels as WT (left panel) $\left(n=6-12\right.$, unpaired t-test $\left.{ }^{* *} p<0.01\right)$.

Data are represented as mean \pm SEM. See Table S3 for details of statistical analysis.

\section{Figure 4. GPR151 is localized at MHb synaptic inputs to IPN}

(A) Schematic of the brain area dissected for EM. (IPR: rostral, IPL: lateral, IPI: intermediate, IPC: central subnucleus of the IPN). 
(B) Representative micrograph showing GPR151 immunogold particles at the presynaptic terminals of WT mice. The presynaptic terminal is delineated in blue. Red arrows point to GPR151 nanogold particles located over the active zone (AZ) or at the membrane. In addition to synaptic vesicles (SV), the terminals contained dense core vesicles (DCV). Scale bar: $500 \mathrm{~nm}$. (mit: mitochondria).

(C) Absence of GPR151 immunogold particles in Gpr151-KO mice. Scale bar: $500 \mathrm{~nm}$.

(D-H) WT and Gpr151-KO mice show no significant differences in synaptic terminal area, length of the active zone and synaptic vesicle diameter, density, and distance to the $A Z$ ( $\mathrm{n} \geq 45$, unpaired t-test, Table S3).

(I-K) Representative micrographs showing GPR151 immunogold particles at the AZ (I), SVs (J) and DCV (K). Scale bar: $100 \mathrm{~nm}$.

(L) Quantitative analysis shows that within a synaptic terminal, GPR151 is mostly at the membrane $(73.63 \% \pm 2.54)$ but can also be found at the $A Z(5.19 \% \pm 1.33)$ and, in association with SV $(9.66 \% \pm 1.890)$; DCV is $(1.39 \% \pm 0.51)$; and other structures $(10.11 \% \pm$ 1.95) ( $n=50$ terminals, total of 999 particles). Data are represented as mean \pm SEM.

(M) Transversal section of an habenular axon showing GPR151 immunogold particles at the plasma membrane and along microtubules. Scale bar: $100 \mathrm{~nm}$.

(N-O) Immunogold particles for GPR151 (12 nm, arrows) and VGLUT1 (6 nm, arrowheads) are localized at presynaptic membranes and SVs in single $(N, O)$ and double $(P, Q)$ postembedding EM experiments. Scale bar: $100 \mathrm{~nm}$.

Data are represented as mean \pm SEM. See Table S3 for details of statistical analysis.

\section{Figure 5. GPR151 contributes to synaptic plasticity}

(A) Chat-ChR2-EYFP mice were crossed to Gpr151-KO for optogenetic recordings. Angled brain sections showing ChR2 (green) and GPR151 (red) in MHb-FR-IPN axis in Chat-ChR2 
and loss of GPR151 signal in Chat-ChR2xGpr151-KO. Chat-ChR2 terminals in the IPN were optogenetically stimulated and postsynaptic IPR neurons were recorded. Scale bar: $100 \mu \mathrm{m}$.

(B) Voltage clamp recordings showing the fidelity of the synaptic responses to blue laser stimulation at $20 \mathrm{~Hz}$ (5 ms pulse width).

(C) Average synaptic fidelity was calculated as the percentage of light pulses that lead to successful transmissions for every second during $1 \mathrm{~min}$ at $20 \mathrm{~Hz}$ stimulation. ChatChR2xGpr151-KO mice (red) showed higher synaptic fidelity compared to Chat-ChR2 mice (black) (n=8-9, RM 2-way ANOVA, $\left.F_{\text {genotype }}(1,15)=6.707, p=0.0205\right)$.

(D) Viral re-expression of GPR151 in the MHb of Chat-ChR2xGpr151-KO mice (blue) decreases synaptic fidelity compared to Chat-ChR2xGpr151-KO mice injected with control virus $(r e d)\left(n=4-10, R M 2-\right.$ way ANOVA, $\left.F_{\text {genotype }}(1,12)=8.379, p=0.0135\right)$.

(E) Synaptic strength measured as the amplitude of the first blue light evoked EPSC is reduced in Chat-ChR2xGpr151-KO mice (red) compared to Chat-ChR2 mice (black) and partially restored by viral re-expression of GPR151 (blue) ( $n=8-16$, one-way ANOVA, Tukey's multiple comparisons test ${ }^{* *} \mathrm{p}=0.0096$ WT vs KO).

(F) Example traces of paired pulse ratio (PPR) recordings in Chat-ChR2 (black) ChatChR2xGpr151-KO (red) mice. The amplitude of the optically evoked EPSCs is reduced in KO as shown in (E) but the paired pulse ratio (PPR) was similar between genotypes $(n=17-16$, unpaired t-test, $p=0.28$ ).

(G-H) Example traces of PPR after vehicle or nicotine (I) Nicotine-induced decreases in PPR were absent in Chat-ChR2xGpr151-KO mice ( $\mathrm{n}=17-8$, Kruskall Wallis test, Dunn's multiple comparisons test $\left.{ }^{*} \mathrm{p}<0.05\right)$

Data are represented as mean \pm SEM. See Table S3 for details of statistical analysis.

Figure 6. GPR151 couples to the G-alpha inhibitory subunit $\mathrm{Ga}_{01}$ and coimmunoprecipitates with pre-synaptic regulators. 
(A) Outline of the co-immunoprecipitation (co-IP) and Mass Spectrometry (MS) experiments ( $n=5$ biological replicates for WT and $n=5$ for KO. For each replicate, 15 IPN were dissected). (B) Volcano plot of proteins that co-IP with GPR151 from the IPN of WT mice (right) and Gpr151-KO (left) IPN samples. Log2 ratios are plotted against the adjusted negative $\log 10 P$ values. Significant different proteins $(p<0.05)$ found in WT samples are labeled in red. The size of the circles indicates an estimate of protein amount. $P$ values were obtained using Student t-test. As expected GPR151 was the most enriched protein in the IP fraction of WT mice and was not present in KO mice. Other co-IP proteins include $\mathrm{Ga}_{01}$, ATPases and SV2A. Control proteins not enriched in WT $(\log 2<1)$ include $\beta_{1}$, ACTB1 and ACTA2 indicated in grey.

(C-D) Western blot confirmation that GPR151 IPs with GPR151 in WT mice (double arrow) and that $\mathrm{Ga}_{01}$ (arrowhead) but not $\mathrm{Ga}_{\mathrm{s}}$ IPs with GPR151 in WT mice (IP GPR151 WT: $1 \pm 0$, KO: $0.03 \pm 0.01, n=5$, unpaired t-test $p<0.0001$; IP Gao1/2 WT: $1 \pm 0, K O: 0.31 \pm 0.09, n=5$, unpaired t-test $p<0.0001$; Input Gao1/2 WT: $1 \pm 0$, KO: $0.98 \pm 0.05, n=5$, unpaired t-test $\mathrm{p}=0.84)$. Data are represented as mean \pm SEM.

\section{Figure 7. GPR151 regulates cAMP levels}

(A) Knockdown of Gpr151 in IPN after injection of LV-shRNA-GFP in the MHb of WT mice in comparison to mice injected with LV-scramble. Scale bar: 100um.

(B) Gpr151-KO and shRNA-injected mice have higher cAMP levels in the IPN than WT and LV-scramble control mice (KO vs WT: $n=15$, unpaired t-test $p=0.0016$, shRNA3 vs scramble $n=8-6$, unpaired t-test $p=0.07)$.

(C) Forskolin increases the amplitude of light evoked EPSCs in Chat-ChR2 neurons $(n=16-6$, one-way ANOVA, Bonferroni's multiple comparison test ${ }^{*} p=0.0149$ ), but has no effect in Chat-ChR2xGpr151-KO neurons ( $\mathrm{n}=11-4$, one-way ANOVA, Bonferroni's multiple comparison test $p>0.99$ ). 
(D) The signal peptide of the 5HT3 receptor and a SNAP-tag encoding region were used to express and monitor expression of human GPR151 in stable cell line. Staining with the cell impermeable SNAP dye 549 shows GPR151 expression at the surface in HEK293 stable cell clone.

(E-F) LANCE cAMP competition assay in HEK293 (E) and CHO-K1 cells (F) under forskolin stimulation (log forskolin nM) shows higher cAMP levels in parental control cells (red) than in GPR151-expressing cells (green). Application of Pertussis toxin (PT) that inactivates G-alpha inhibitory subunits including $\mathrm{Ga}_{\mathbf{0} 1}$ (present in CHO-K1 but not in HEK293) decreases the EC50 in CHO-GPR151 but not in HEK293-GPR151 cells ( $n=2$ technical triplicates for each response curve, one-way ANOVA for $\mathrm{EC}_{50}$ values, Bonferroni's multiple-comparison test, ${ }^{* *} p=0.0085$ HEK293-Control vs HEK293-GPR151, $p>0.99$ HEK293-Control vs HEK293Control+PTX, p>0.99 HEK293-GPR151 vs HEK293-GPR151+PTX, ${ }^{* * *}{ }^{*}<0.0001$ CHO-K1Control vs CHO-K1-GPR151, p>0.99 CHO-K1-Control vs CHO-K1-Control+PTX, ${ }^{* * *} p=0.0004$ CHO-K1-GPR151 vs CHO-K1-GPR151+PTX).

Data are represented as mean \pm SEM. See Table S3 for details of statistical analysis. 
A

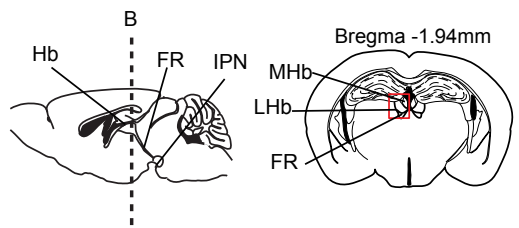

E
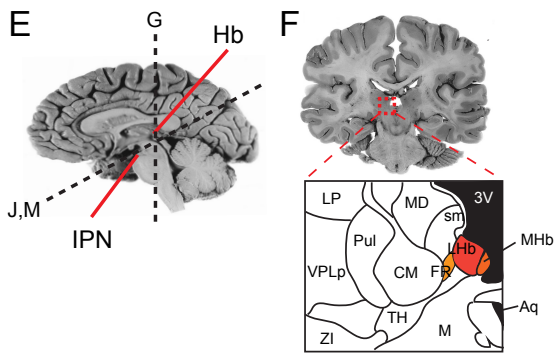

P

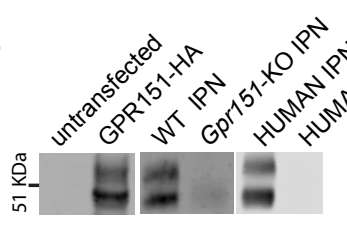

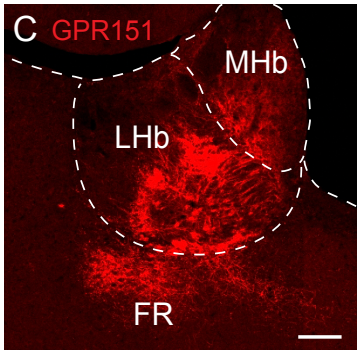
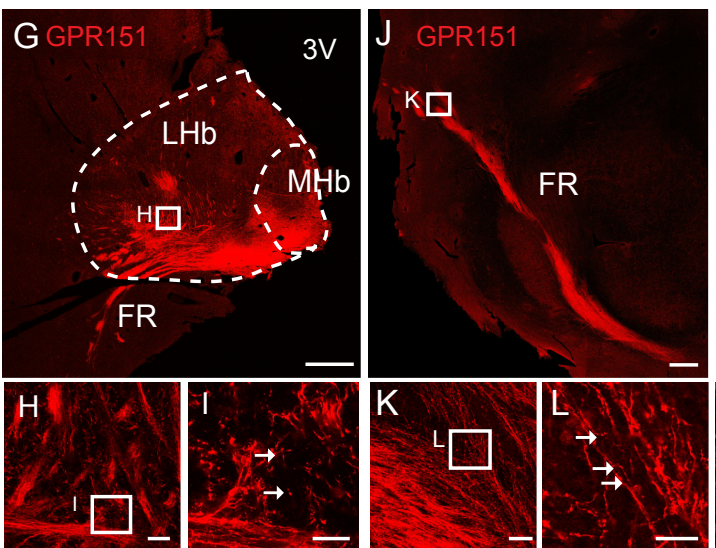

Q

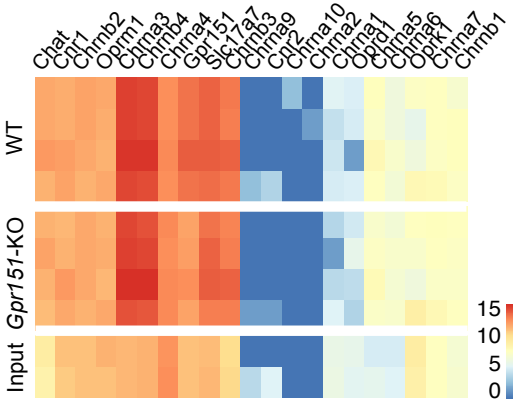

R

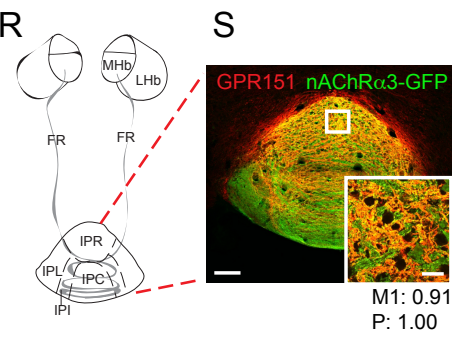

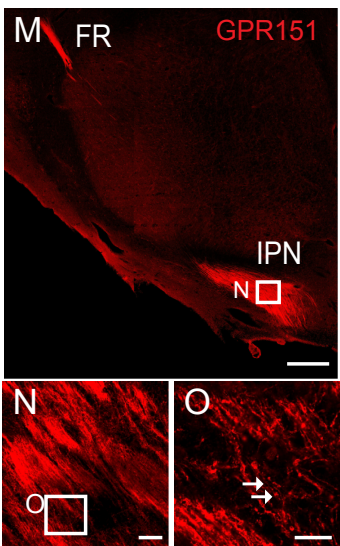

$\mathrm{Hb}$

FR

IPN

T

V
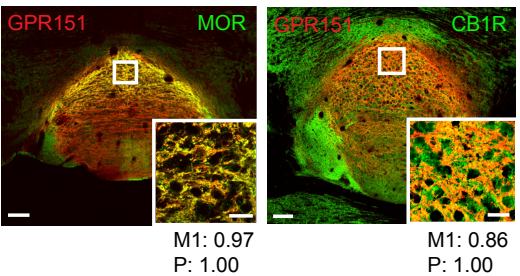


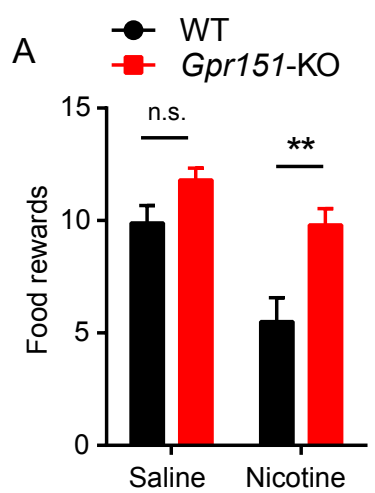

B

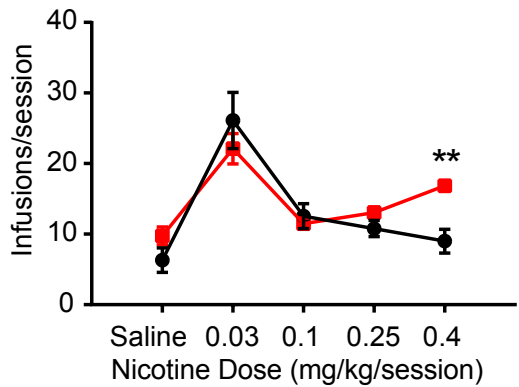

C

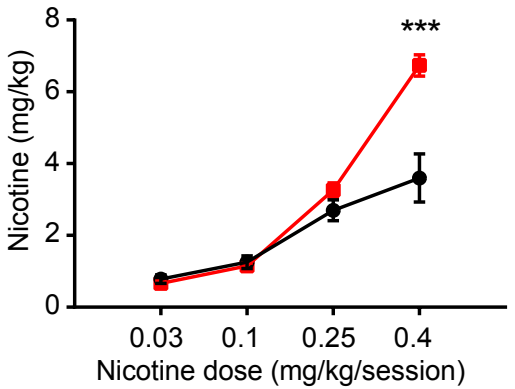

D

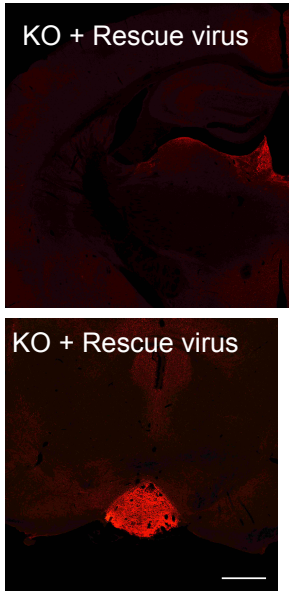

GPR151

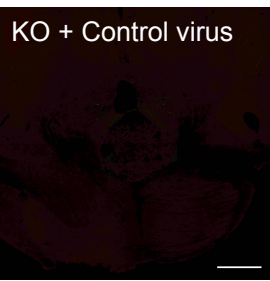

E
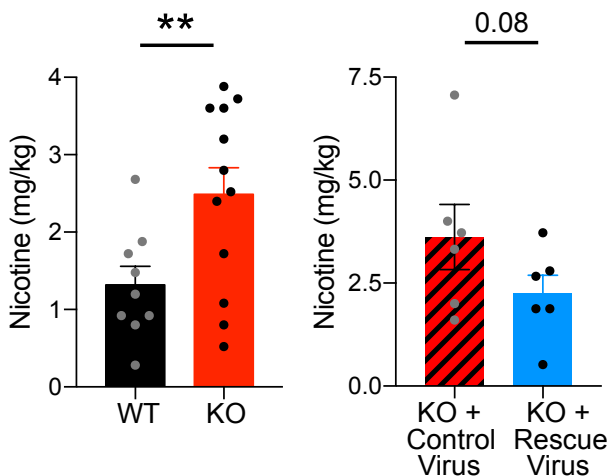

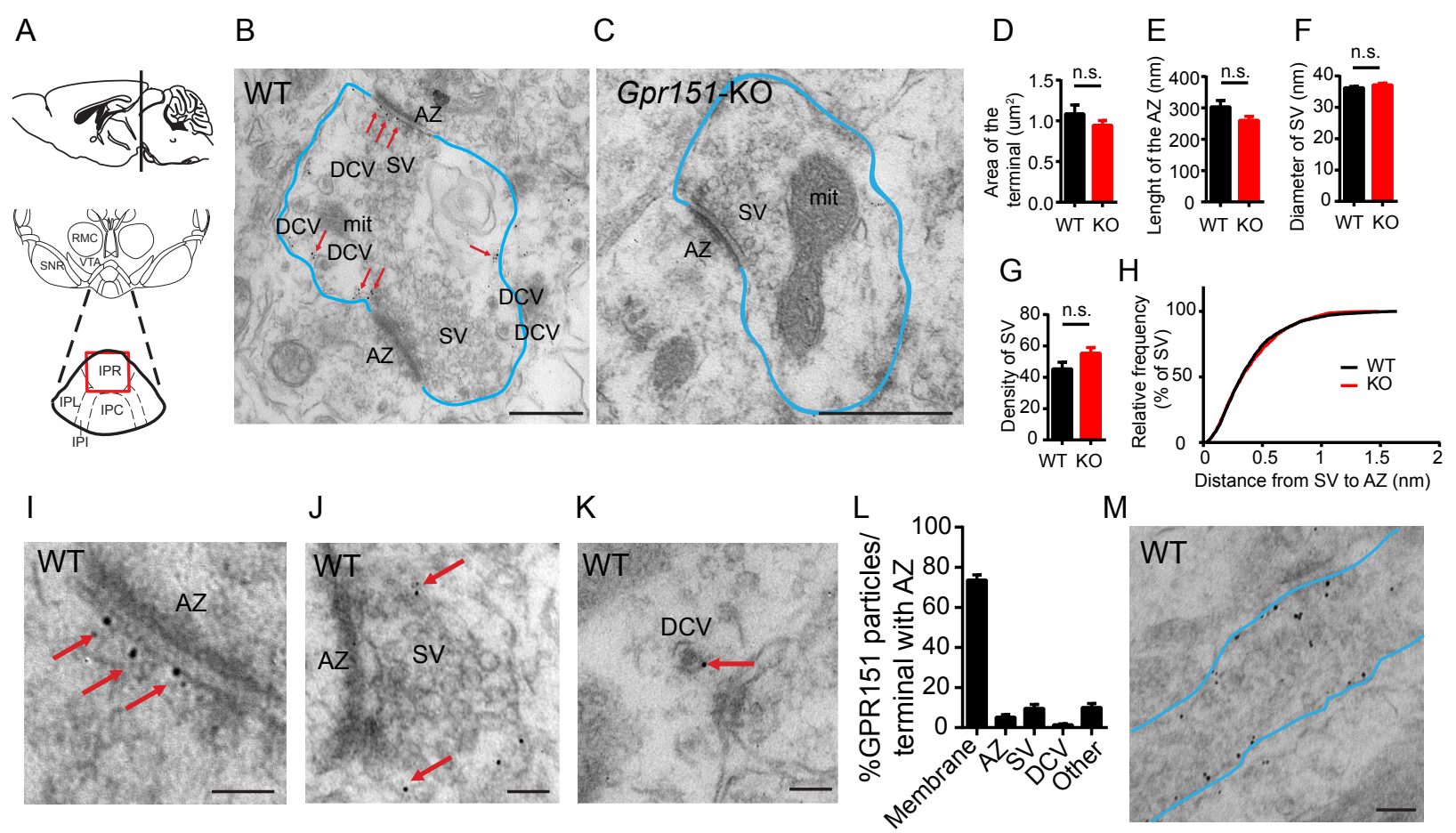

GPR151- $12 \mathrm{~nm}$ gold particles
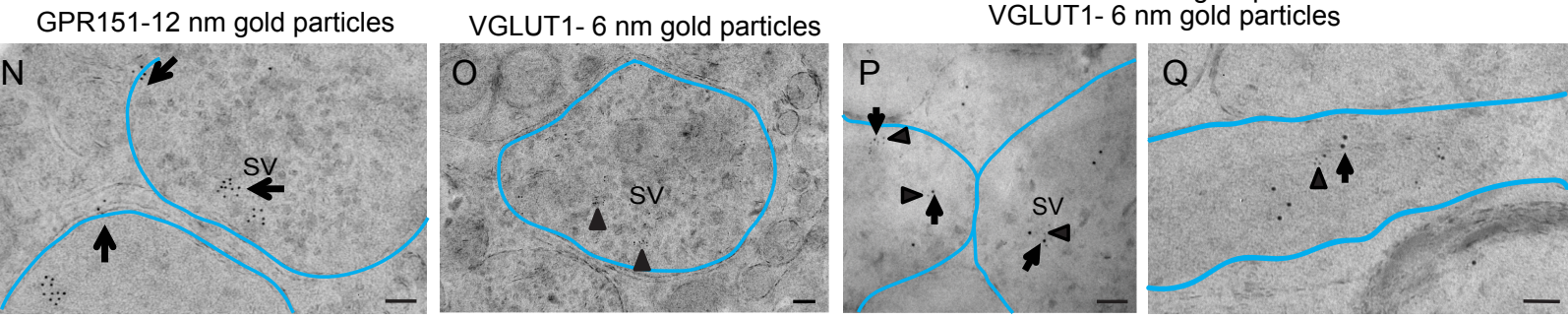


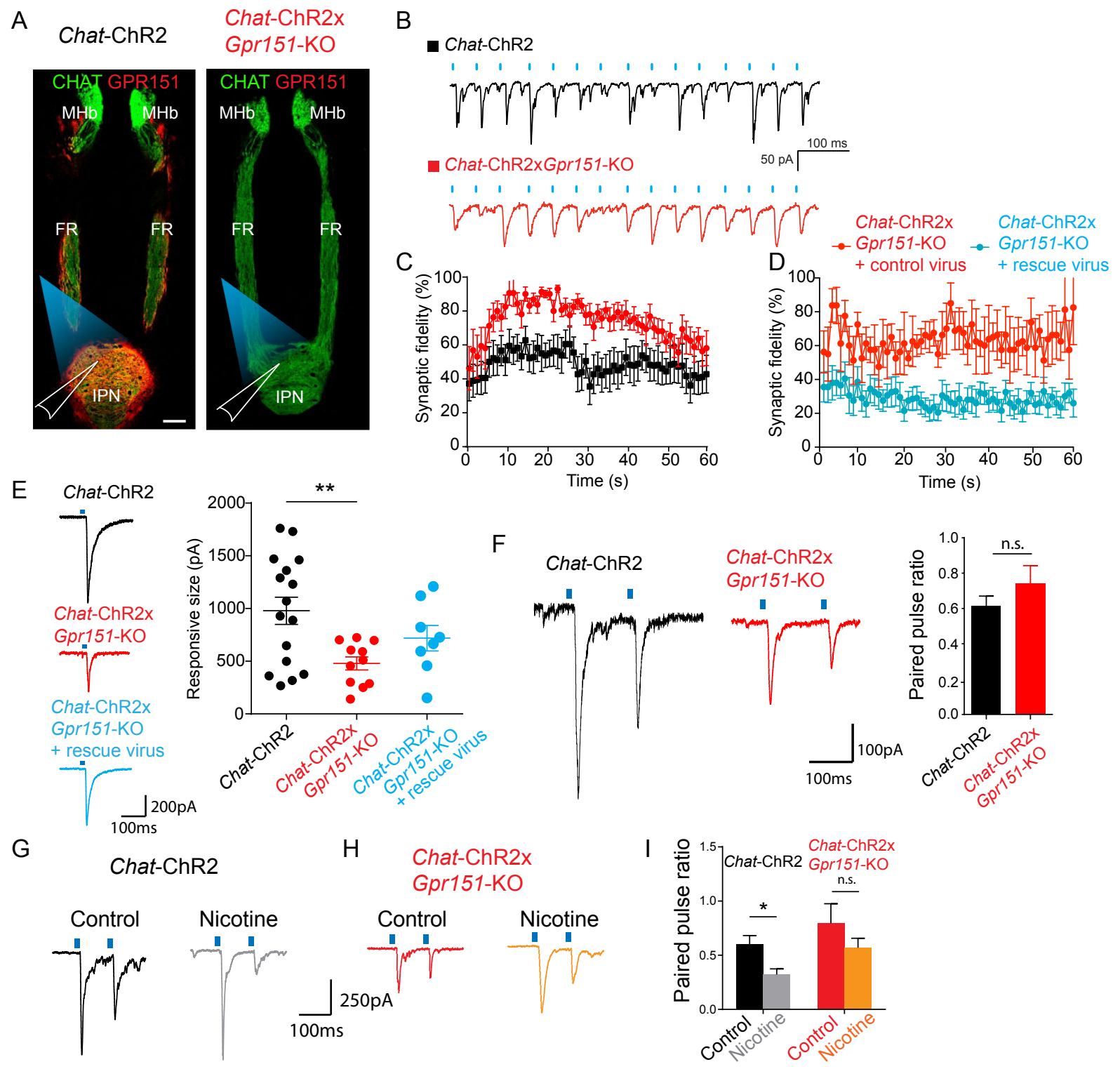


A

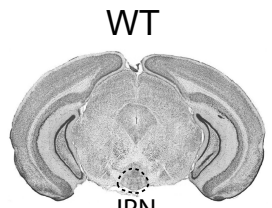

IPN

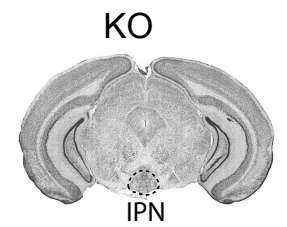

1) Homogeneization

2) IP with anti-GPR151 antibody

3) Elution

4) Trypsin digestion

5) MS analysis (MaxQuant)

C
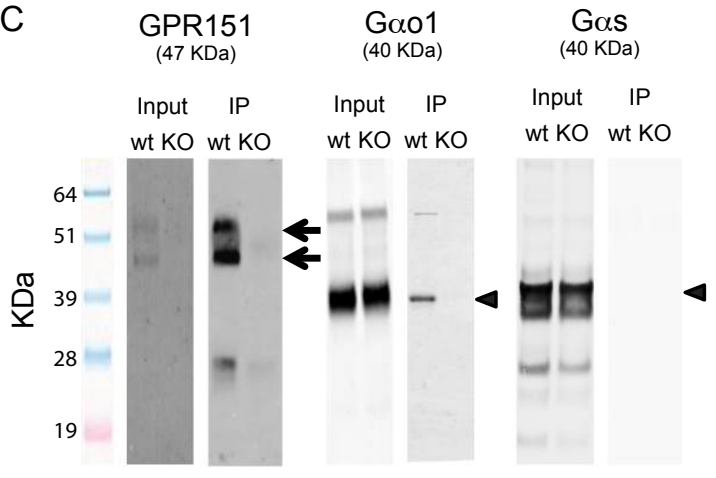

B

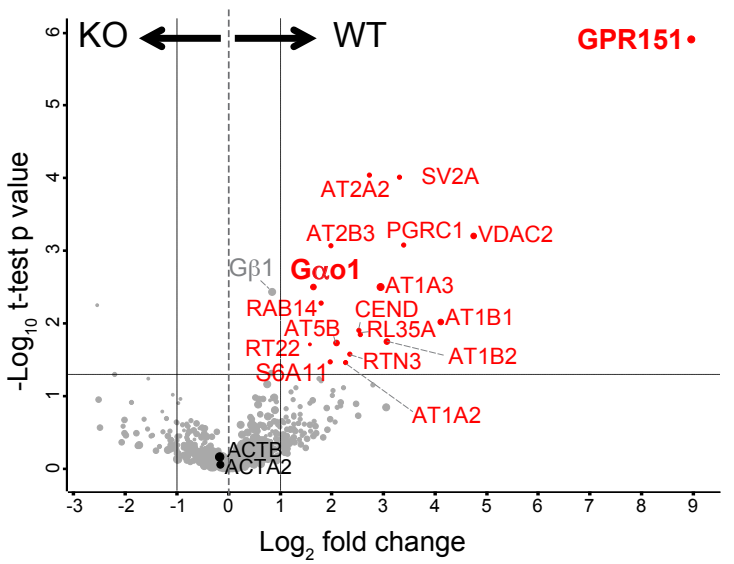

D

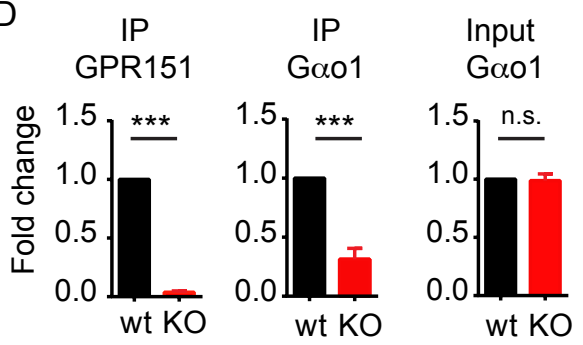



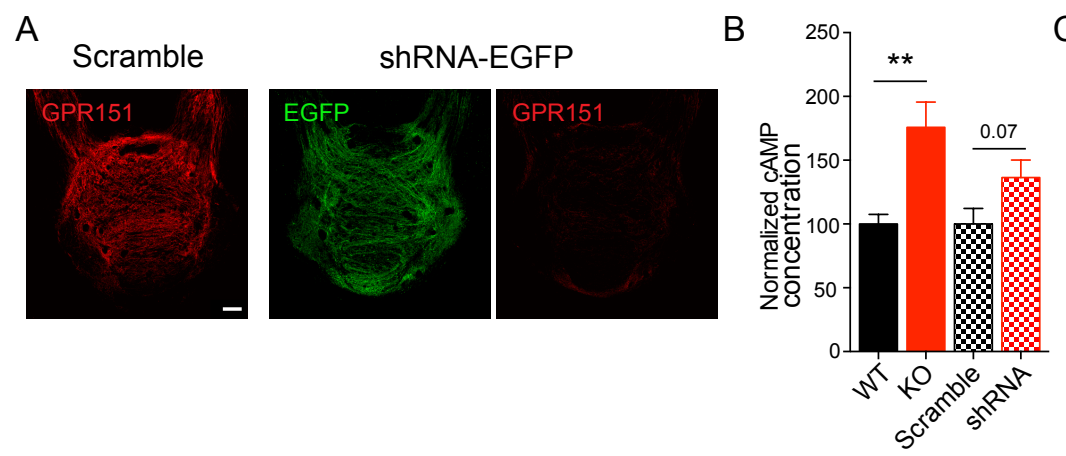

C

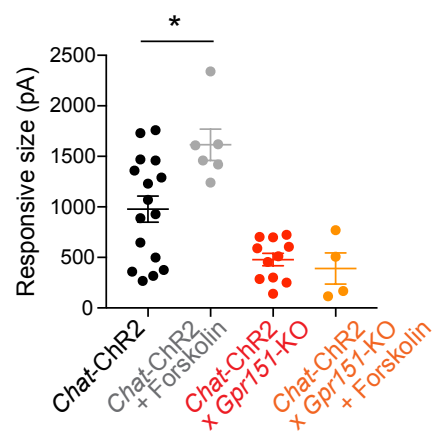

D 5HT3SigP/SNAP/GPR151 E
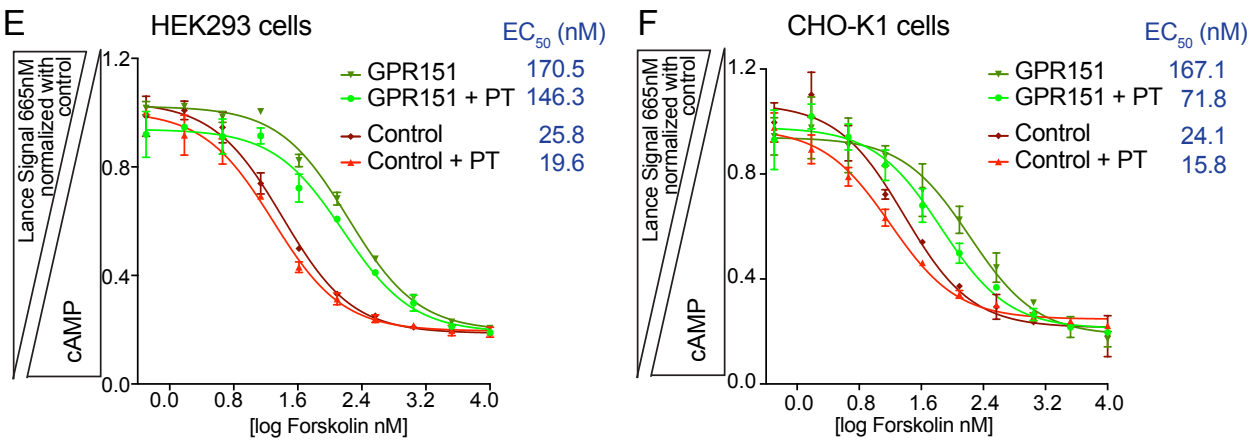